\title{
A POGLUT1 mutation causes a muscular dystrophy with reduced Notch signaling and satellite cell loss
}

\author{
Emilia Servián-Morilla ${ }^{1,2, \dagger}$, Hideyuki Takeuchi ${ }^{3, \dagger}$, , Tom V Lee ${ }^{4, \dagger}$, Jordi Clarimon ${ }^{2,5}$, Fabiola Mavillard ${ }^{2,6}$, \\ Estela Area-Gómez7, Eloy Rivas ${ }^{8}$, Jose L Nieto-González ${ }^{2,6}$, Maria C Rivero ${ }^{2,6}$, Macarena Cabrera- \\ Serrano ${ }^{1,2}$, Leonardo Gómez-Sánchez ${ }^{2,6}$, Jose A Martínez-López ${ }^{2,6}$, Beatriz Estrada9 \\ Celedonio Márquez ${ }^{1}$, Yolanda Morgado ${ }^{10}$, Xavier Suárez-Calvet ${ }^{11,12}$, Guillermo Pita ${ }^{13}$, \\ Anne Bigot ${ }^{14}$, Eduard Gallardo ${ }^{11,12}$, Rafael Fernández-Chacón ${ }^{2,6}$, Michio Hirano, \\ Robert S Haltiwanger ${ }^{3, *}$, Hamed Jafar-Nejad ${ }^{4}$ \& Carmen Paradas ${ }^{1,2,7}$,
}

\begin{abstract}
Skeletal muscle regeneration by muscle satellite cells is a physiological mechanism activated upon muscle damage and regulated by Notch signaling. In a family with autosomal recessive limbgirdle muscular dystrophy, we identified a missense mutation in POGLUT1 (protein 0 -glucosyltransferase 1 ), an enzyme involved in Notch posttranslational modification and function. In vitro and in vivo experiments demonstrated that the mutation reduces $O$ glucosyltransferase activity on Notch and impairs muscle development. Muscles from patients revealed decreased Notch signaling, dramatic reduction in satellite cell pool and a muscle-specific $\alpha$ dystroglycan hypoglycosylation not present in patients' fibroblasts. Primary myoblasts from patients showed slow proliferation, facilitated differentiation, and a decreased pool of quiescent $\mathrm{PAX7}^{+}$ cells. A robust rescue of the myogenesis was demonstrated by increasing Notch signaling. None of these alterations were found in muscles from secondary dystroglycanopathy patients. These data suggest that a key pathomechanism for this novel form of muscular dystrophy is Notch-dependent loss of satellite cells.
\end{abstract}

Keywords muscular dystrophy; Notch; O-glycosylation; POGLUT1;

satellite cell
Subject Categories Development \& Differentiation; Musculoskeletal System DOI 10.15252/emmm.201505815 | Received 2 September 2015 | Revised 22 August 2016 | Accepted 2 September 2016 | Published online 10 October 2016 EMBO Mol Med (2016) 8: 1289-1309

\section{Introduction}

Cell surface glycans are diverse in structure and function and play critical roles in many biological processes including infection, cancer, and development (2009). Broadly speaking, glycans affect the function of proteins to which they are linked by modulating their structure and/or by serving as a direct recognition signal for other proteins (Varki \& Sharon, 2009). Glycans are involved in protein folding, stability, and trafficking and regulate the activity of important signaling pathways such as Notch, WNT, BMP, and Hedgehog (Christian, 2000; Yan \& Lin, 2009; Jafar-Nejad et al, 2010; Takeuchi \& Haltiwanger, 2014). Not surprisingly, aberrant glycosylation leads to a variety of human diseases, the list of which is growing (Freeze et al, 2014).

Protein glycosylation can directly regulate signaling events. A clear example of this is the regulation of the evolutionarily conserved Notch signaling pathway, which is an intercellular

\footnotetext{
1 Neuromuscular Disorders Unit, Department of Neurology, Instituto de Biomedicina de Sevilla, Hospital U. Virgen del Rocío/CSIC/Universidad de Sevilla, Sevilla, Spain

2 Centro de Investigación Biomédica en Red sobre Enfermedades Neurodegenerativas (CIBERNED), Madrid, Spain

3 Department of Biochemistry and Cell Biology, Stony Brook University, Stony Brook, NY, USA

4 Department of Molecular and Human Genetics, Baylor College of Medicine, Houston, TX, USA

5 Memory Unit, Department of Neurology and Sant Pau Biomedical Research Institute, Hospital de la Santa Creu i Sant Pau, Universitat Autònoma de Barcelona, Barcelona, Spain

6 Department of Medical Physiology and Biophysics, Instituto de Biomedicina de Sevilla, Hospital U. Virgen del Rocío/CSIC/Universidad de Sevilla, Sevilla, Spain

7 Department of Neurology, Columbia University Medical Center, New York, NY, USA

8 Department of Pathology, Instituto de Biomedicina de Sevilla, Hospital U. Virgen del Rocío/CSIC/Universidad de Sevilla, Sevilla, Spain

9 Centro Andaluz de Biología del Desarrollo (CABD), Universidad Pablo Olavide, Sevilla, Spain

10 Department of Neurology, Hospital U. Valme, Sevilla, Spain

11 Laboratori de Malalties Neuromusculars, Institut de Recerca de HSCSP, Universitat Autònoma de Barcelona (UAB), Barcelona, Spain

12 Centro de Investigación Biomédica en Red sobre Enfermedades Raras (CIBERER), Barcelona, Spain

13 Human Genotyping Unit-CeGen, Spanish National Cancer Research Centre, Madrid, Spain

14 UPMC Univ Paris 06, INSERM UMRS974, CNRS FRE3617, Center for Research in Myology, Sorbonne Universités, Paris, France

*Corresponding author. Tel: +34 955923045; Fax: +34 955013536; E-mail: cparadas@us.es

†These authors contributed equally to this work

"Present address: Complex Carbohydrate Research Center, The University of Georgia, Athens, GA, USA
} 
communication mechanism widely used during animal development (Hori et al, 2013). The extracellular domain of the Notch receptor is modified with $\mathrm{O}$-fucose and $\mathrm{O}$-glucose glycans (Moloney et al, 2000). The structures of these glycans change in a tissue-specific and developmentally regulated manner (Johnston et al, 1997), and altering Notch glycosylation dramatically affects its activity (Stanley \& Okajima, 2010; Takeuchi \& Haltiwanger, 2010). One of the cell types whose regulation by Notch signaling has been intensely studied in recent years is the muscle-specific adult stem cell called the satellite cell (SC) (Mourikis \& Tajbakhsh, 2014). SCs reside between the basal lamina and sarcolemma of myofibers (Mauro, 1961) and are the primary contributors to skeletal muscle growth and repair (Collins et al, 2005). Despite continuous regeneration, pools of SC are maintained in healthy muscle. This is accomplished through asymmetric cell divisions of SC, which give rise both to self-renewing SC and to committed myogenic progenitors that differentiate (Kuang et al, 2007; Sacco et al, 2008). In this process, the Notch pathway is key for maintaining quiescence in SC and for homing of SC-derived myoblasts (Bjornson et al, 2012; Brohl et al, 2012). A recent report indicates that mice in which Notch signaling is specifically blocked in SCs exhibit a decrease in the number of SCs and histological features of muscular dystrophy even upon normal daily activity (Lin et al, 2013), suggesting that Notch-mediated maintenance of an active SC pool is essential for repairing muscle damage caused by regular activity and for maintaining healthy muscle. Muscular dystrophies are inherited disorders characterized by progressive weakness due to skeletal muscle degeneration, due to mutations in a growing list of responsible genes (Chandrasekharan \& Martin, 2010; Rahimov \& Kunkel, 2013). However, to date, no primary molecular defects in SC or Notch pathway components have been identified in human muscular dystrophies.

Mutations in several genes disrupt various aspects of the dystrophin-associated glycoprotein complex, which links the cytoskeleton to the extracellular matrix in muscle (Chandrasekharan \& Martin, 2010). A key component of this complex is dystroglycan (Ervasti et al, 1990), a transmembrane protein essential for normal basement membrane development and muscle maintenance. Dystroglycan comprises a transmembrane $\beta$-subunit non-covalently linked to an extracellular $\alpha$-subunit containing a mucin-like domain, which is modified with numerous O-linked glycans (Barresi \& Campbell, 2006). The $\beta$-subunit is linked to the actin cytoskeleton, and the $O$-linked glycans on the $\alpha$-subunit are critical for its binding capacity to extracellular matrix proteins such as laminin and agrin (Ervasti \& Campbell, 1993; Michele et al, 2002). Mutations in dystroglycan itself result in the primary dystroglycanopathies (Henry \& Campbell, 1998; Cote et al, 1999; Hara et al, 2011; Willer et al, 2014; Riemersma et al, 2015; Kanagawa et al, 2016). Secondary dystroglycanopathies are caused by interruption of $\alpha$-dystroglycan-ligand interactions due to mutations in a growing list of genes [currently eighteen (Bonnemann et al, 2014)] encoding glycosyltransferases and accessory proteins responsible for $\alpha$-dystroglycan's extensive posttranslational modifications (Muntoni et al, 2011; Inamori et al, 2012; Yoshida-Moriguchi et al, 2013). These include protein $O$-mannosyltransferase 1 (POMT1) and POMT2, which add $O$-linked mannose to $\alpha$-dystroglycan (Manya et al, 2004; Willer et al, 2004), and like-acetylglucosaminyltransferase (LARGE), which is responsible for the addition of repeating xylose-glucuronic acid units on the $O$-mannosyl glycans on $\boldsymbol{\alpha}$-dystroglycan (Inamori et al, 2012). The dystroglycan gene (DAG1) is expressed in SCs (Cohn et al, 2002), but glycosylated $\alpha$-dystroglycan is not required by myogenic cells during proliferation (Awano et al, 2015), a notion supported by the observation that trace amounts of glycosylated $\alpha$-dystroglycan are found in $\mathrm{C} 2 \mathrm{C} 12$ and human primary myoblasts during proliferation. Cultures of freshly isolated muscle fibers suggest that glycosylation of $\alpha$-dystroglycan is needed for proliferation of SCs in vivo, but it is not necessary when SCs are removed from their niche (Ross et al, 2012). The transition to larger glycans occurs rapidly after differentiation is induced, and a direct correlation exists between LARGE-dependent extension of $O$-glycans on $\alpha$-dystroglycan and its function as an extracellular matrix receptor (Goddeeris et al, 2013). Despite extensive studies regarding the genetics of dystroglycanopathies, the responsible gene is still unknown in a significant number of patients; thus, mutations in additional genes will likely be implicated in this group of diseases.

Here, we report a homozygous missense mutation (D233E) in the protein $O$-glucosyltransferase 1 gene, POGLUT1, in four siblings with autosomal recessive limb-girdle muscular dystrophy. POGLUT1 is the sole enzyme that directly adds $O$-glucose to a distinct serine residue of epidermal growth factor-like (EGF) repeats containing a CXSX(P/A)C consensus sequence (Rana et al, 2011), many of which are found in Notch extracellular domains (Fernandez-Valdivia et al, 2011; Rana et al, 2011). The D233E mutation dramatically reduces the $O$-glucosyltransferase activity of POGLUT1, leading to impaired Notch signaling and a dramatic decrease in the number of SCs in adult muscles. Transgenic experiments in Drosophila indicate that D233E impairs the ability of human POGLUT1 to rescue the muscle phenotype caused by the loss of fly Poglut 1 activity. Patient muscles show $\alpha$-dystroglycan hypoglycosylation and decreased binding to laminin, but normal binding to agrin and normal basement membrane structure. Moreover, unlike other dystroglycanopathies, patient fibroblasts exhibit normal $\boldsymbol{\alpha}$-dystroglycan glycosylation and laminin binding. Together, our findings indicate that exhaustion of the SC pool plays a primary role in this novel form of muscular dystrophy.

\section{Results}

\section{Clinical and radiological findings}

A consanguineous family from southern Spain comprises 17 individuals spanning three generations (Fig 1A). Four out of five siblings from generation II presented a phenotype consistent with a limb-girdle muscular dystrophy. Specifically, the patients exhibited muscle weakness predominantly in the proximal lower limbs, with onset during the third decade. The disease course was progressive, leading to scapular winging and wheelchair confinement. For more extended clinical data regarding this family, see the Appendix Information, Appendix Fig S1, and Appendix Tables S1 and S2. Serum creatine kinase level was normal in three patients and mildly elevated in one (Appendix Table S1). Muscle biopsies from all four affected siblings revealed histological features ranging from very mild myopathic changes to classic dystrophic pathology (Fig 1A). Proteins typically affected in myopathies displayed normal expression in muscle, except for a reduction in $\alpha$-dystroglycan 
A

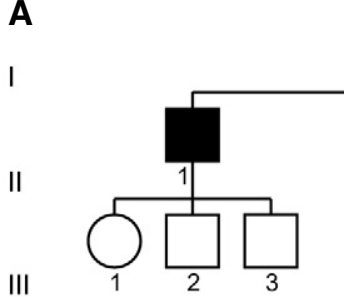

III Patient II.1
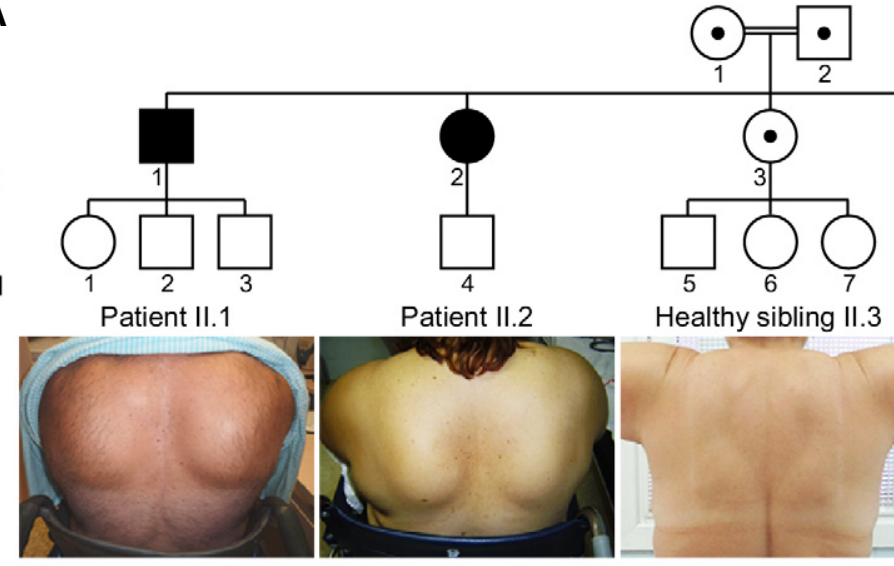
Healthy sibling II.3
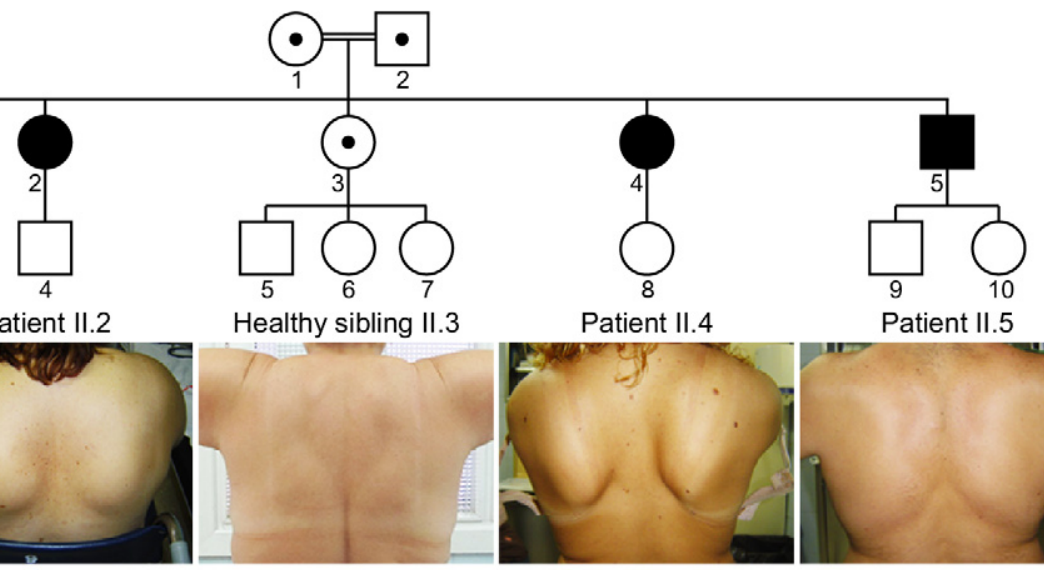

Patient II.5
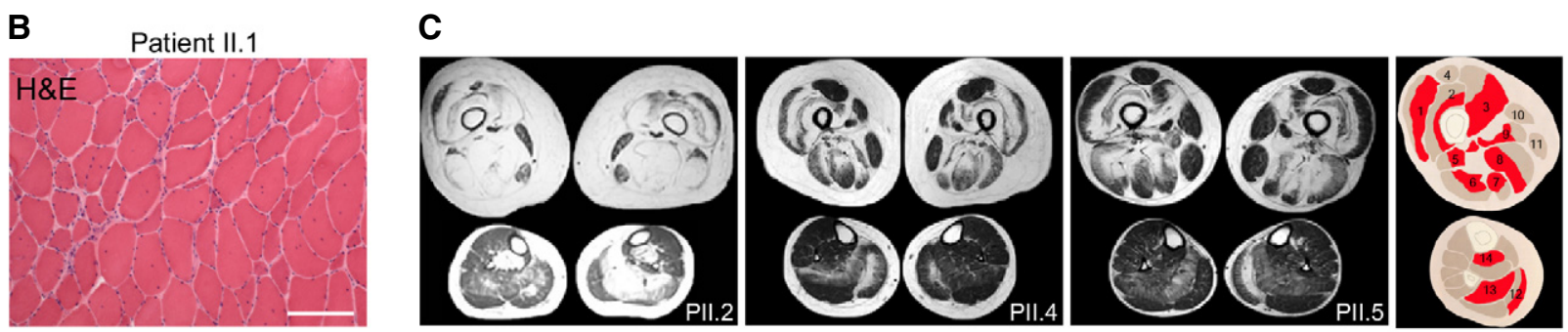

Figure 1. POGLUT1 missense mutation in a family with a limb-girdle muscular dystrophy.

A The family pedigree, where circles denote female members, squares male members, solid symbols affected members, and white symbols asymptomatic members with normal physical exam; the dots indicate heterozygous carriers, and double line denotes a consanguineous marriage. The pictures show scapular winging, which is a consistent clinical sign in affected individuals.

B Hematoxylin and eosin staining (H\&E) of skeletal muscle from patient II.1 shows histological features of moderate-to-severe dystrophic pattern. Scale bar, 50 $\mu$ m.

C T1-weighted MRI axial images at thigh and calf levels show that the fatty degeneration is more prominent in thigh muscles, equally affecting posterior and anterior compartments, with relative sparing of the rectus femoris, sartorius, and gracilis muscles until late stages (4, 10, and 11 , respectively). Strikingly, the fatty tissue is located in the internal parts of almost all the affected muscles in thigh (1, 2, 3, 5-9), while the external regions are spared. At calf level, only the gastrocnemius medialis muscle (12) shows this pattern, while the soleus (13) is diffusely involved. Patient II.2 (PII.2) shows late-stage thigh muscles with an unusual involvement of the tibialis posterior muscle (14) in the lower leg.

(Appendix Fig S2). Muscle magnetic resonance imaging (MRI) of the legs revealed a striking pattern of muscle involvement (Fig 1C), with early fatty replacement of internal regions of thigh muscles that spared external areas. This "from inside-to-outside" mode of fatty degeneration progressed over the years and did not match the distribution patterns typically associated with other forms of muscular dystrophies (Appendix Information and Appendix Figs S3 and S4).

\section{Expression and functional modification of $\alpha$-dystroglycan in patients}

Given the key role played by aberrant $\alpha$-dystroglycan glycosylation and function in a subset of muscular dystrophies and because of the observed decrease in $\alpha$-dystroglycan levels in patient muscles, we examined the glycosylation status and ligand-binding ability of $\alpha$-dystroglycan in our patients. Immunofluorescence staining of frozen cross sections from skeletal muscle biopsy with an antibody against glycosylated $\alpha$-dystroglycan [IIH6 (Ervasti \& Campbell, 1991)] revealed a variable reduction in the glycosylated form of $\alpha$-dystroglycan at the sarcolemma in patients, while antibodies against $\alpha$-dystroglycan core protein, $\beta$-dystroglycan, and laminin $\alpha 2$ showed normal staining (Fig 2A and Appendix Fig S5A). In agreement with this observation, Western blots showed a reduction in $\alpha$-dystroglycan glycosylation in patient muscle, accompanied by a mild decrease in the molecular weight of glycosylated $\alpha$-dystroglycan compared with controls. To examine whether decreased $\alpha$-dystroglycan glycosylation affected binding to ligands, we performed a ligand overlay assay. As shown in Fig 2B, the lamininbinding activity was diminished in muscle. However, the agrinbinding activity to the patients' muscle extracts showed no difference compared with controls (Fig 2B). Moreover, in skin fibroblasts from patients, the level of both functional $\alpha$-dystroglycan glycosylation, examined by Western blot and flow cytometry (Stevens et al, 2013b), and its ability to bind laminin (Fig 2B and Appendix Fig S5B) were normal, unlike known secondary dystroglycanopathies, which usually result in decreased functional $\alpha$-dystroglycan glycosylation in both muscle and the skin fibroblasts (Willer et al, 2012; Carss et al, 2013; Stevens et al, 2013a). Finally, although basement membrane defects are commonly observed in dystroglycanopathies (Yamamoto et al, 1997; Goddeeris et al, 2013), transmission electron microscopy showed normal muscle ultrastructure in patients, with no alterations in basement membrane compaction (Fig 2C and Appendix Fig S5C). 
A
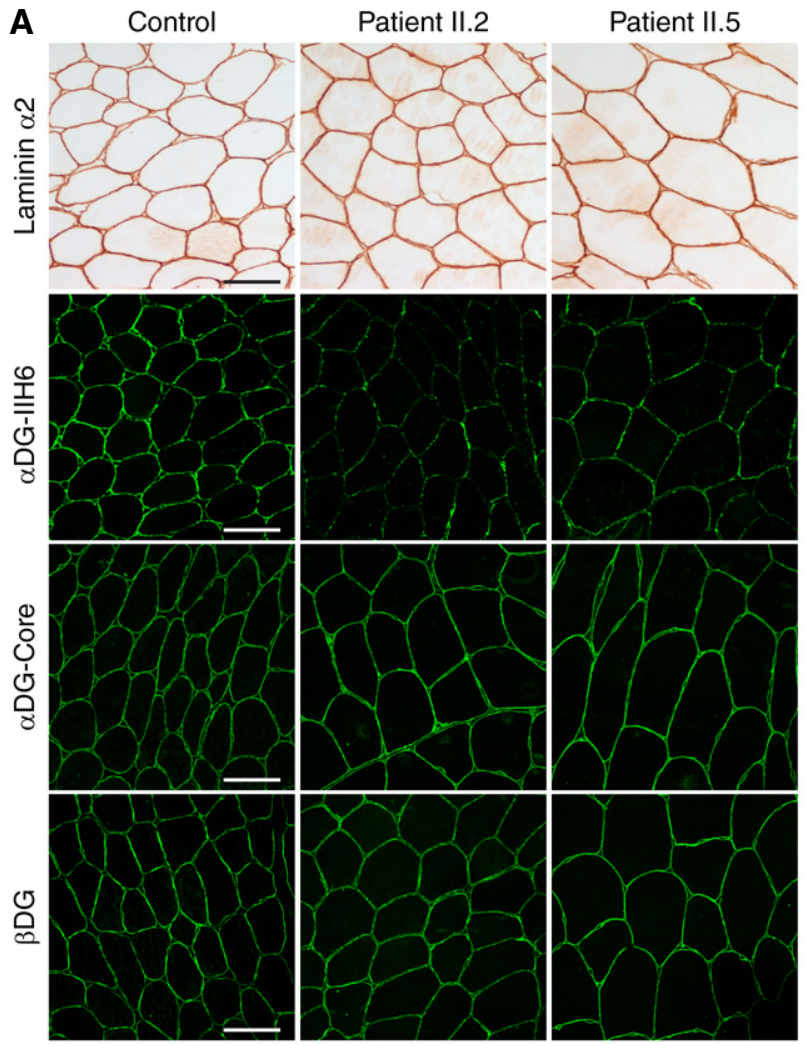

C
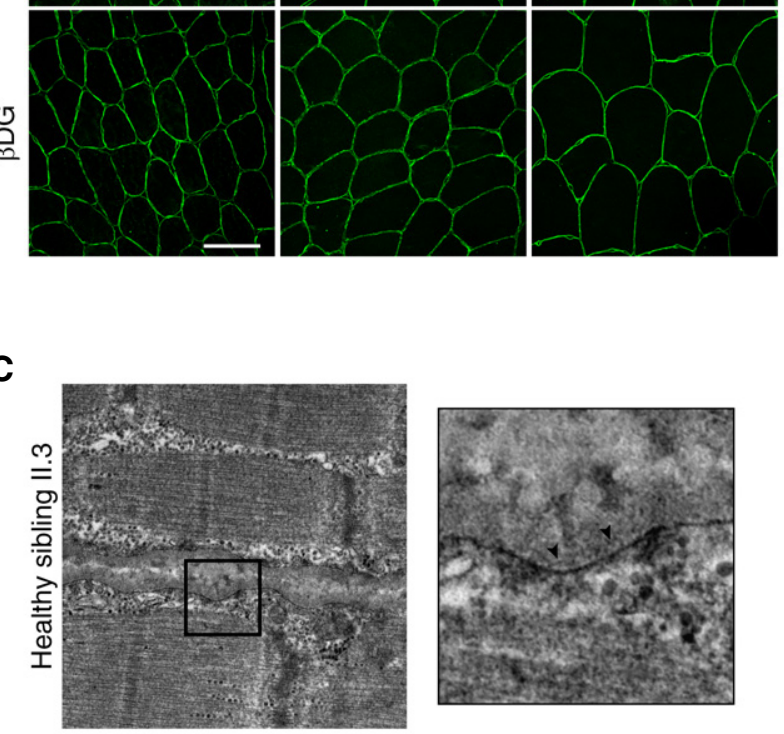

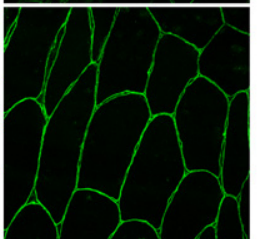

B

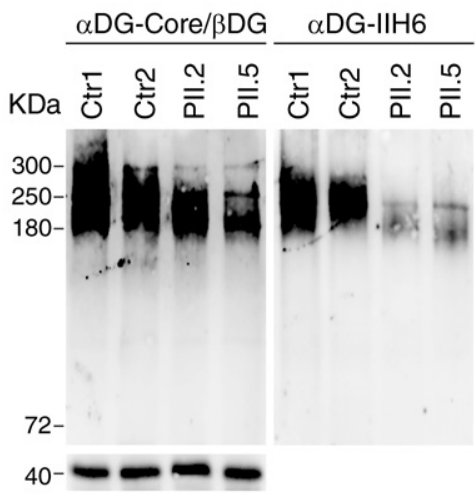

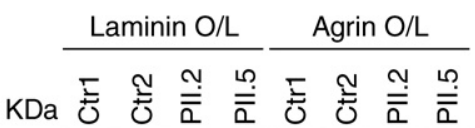

Muscle

Muscle

Fibroblasts

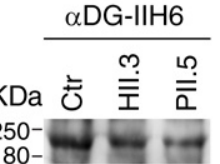

$\alpha \mathrm{DG}-\mathrm{Core} / \beta \mathrm{DG}$

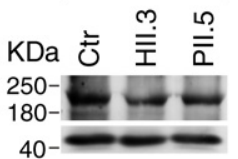

Fibroblasts

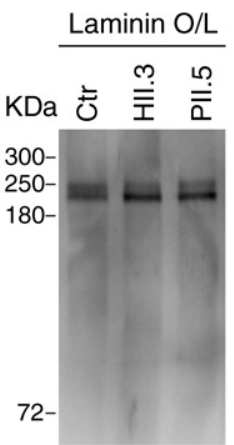

Figure 2. A muscle-specific decrease in $\alpha$-dystroglycan glycosylation and laminin binding upon POGLUT1 mutation.

A Muscle sections show variable labeling using an antibody against glycosylated $\alpha$-dystroglycan ( $\alpha \mathrm{DG}-\mathrm{IIH} 6$ ), whereas labeling using antibodies against $\alpha$-dystroglycan core protein ( $\alpha \mathrm{DG}$-Core), $\beta$-dystroglycan ( $\beta D \mathrm{DG})$, and laminin- $\alpha 2$ is similar to control (scale bar, $100 \mu \mathrm{m}$ ).

B Western blots and ligand overlay (O/L) of wheat germ agglutinin-enriched muscle and fibroblasts lysates from PII.2, PII.5, the healthy sibling (HII.3), and healthy controls (Ctr, Ctr1, and Ctr2) (muscle: $250 \mu \mathrm{g}$ protein/lane, fibroblasts: $800 \mu \mathrm{g} / \mathrm{lane}$ ). In muscle, expression of $\alpha \mathrm{DG}-\mathrm{IIH} 6$ is reduced, but the $\alpha \mathrm{DG}$-Core shows similar expression as controls, with a slight reduction in molecular weight; laminin overlay assay detected some binding activity but was diminished compared with controls, whereas agrin-binding activity showed no difference with controls. In fibroblasts, both $\alpha \mathrm{DG}-\mathrm{IIH} 6$ and $\alpha \mathrm{DG}$-Core expression and laminin-binding activity were normal, suggesting that unlike other muscular dystrophies, $\alpha$-dystroglycan glycosylation defect is muscle specific in POGLUT1 patients.

C No ultrastructural alterations are observed in muscle by electron microscopy (6-7 fields were captured from PII.1, PII.3, and PII.5). Note normal basement membrane compaction (arrowheads). Original magnification: $\times 26,700$.

Source data are available online for this figure.

These observations suggest that although a partial decrease in $\alpha$-dystroglycan functional glycosylation is detected in muscle from our patients, decreased $\alpha$-dystroglycan glycosylation is unlikely to be caused by the same pathomechanism seen in secondary dystroglycanopathies and that a mutation in a gene other than the enzymes directly involved in $\alpha$-dystroglycan glycosylation might underlie this disease.

\section{D233E mutation in POGLUT1}

Homozygosity mapping in DNA samples through genomewide genotyping arrays containing $\sim$ one million single nucleotide polymorphisms (SNPs) revealed a unique ( $>500 \mathrm{~kb}$ ) region of homozygosity identical by descent in the four affected siblings and absent in their 
healthy sister (Fig 3A). This 14.6 megabase region on chromosome 3q13.13-q21.2, defined by SNP markers rs13081374 and rs2333038, included 112 protein-coding genes (Fig 3B and C). No structural genomic variations were shared by affected family members, thus excluding gross gene dosage change as a cause of disease. We next performed whole exome sequencing of patients II.4, II.5, and their healthy sister II.3 and found a c.699T > G transversion within POGLUT1 (Fig 3D). The polymorphism produces an aspartic-toglutamic substitution at amino acid residue 233 (p.D233E), which passed all possible filtering, segregated with the disease, and consequently was suspected to be the causal genetic alteration.

Significantly, POGLUT1 is located within the 3q13.13-q21.2 region of homozygosity in the affected siblings (Fig 3C). The mutation was not found in the Exome Aggregation Consortium (ExAC) database (http://exac.broadinstitute.org) [accessed in February 2015], thus discarding this genetic variant in 60,698 individuals (36,673 Europeans). In addition, the D233E mutation was further excluded in 919 DNA samples from 57 populations worldwide (Appendix Table S3).

The mutated aspartic acid residue is located in the capsule-associated protein (CAP) 10 domain, 14 amino acids apart from the putative ERD catalytic motif, and is highly conserved across vertebrate species (Fig 3E).

\section{Expression of POGLUT1 ${ }^{\mathrm{D} 233 \mathrm{E}}$ in patients}

We assessed the effect of the D233E mutation on the expression of POGLUT1 in samples from patients. In skeletal muscle, Western blot showed normal protein expression and qRT-PCR assays showed no difference in the POGLUT1 mRNA level compared with controls. In skin biopsy-derived fibroblasts, immunostaining revealed a colocalization of POGLUT1 ${ }^{\text {D233E }}$ with the endoplasmic reticulum, displaying the same pattern as the wild-type POGLUT1 in human control fibroblasts (Appendix Fig S6). These data indicate that the D233E mutation does not affect the expression level or subcellular localization of POGLUT1.

\section{Biochemical assays of D233E mutation in POGLUT1}

We next analyzed the effect of the D233E mutation on the O-glucosyltransferase activity of POGLUT1 by biochemical assays. Wildtype and D233E mutant forms of human-POGLUT1 were transiently expressed in HEK293T cells and purified from the culture media (Appendix Fig S7A). We examined the enzymatic activity of purified proteins toward a known substrate: bacterially expressed epidermal growth factor-like (EGF) repeat from human coagulation factor IX (hFIX). POGLUT1 ${ }^{\text {D233E }}$ mutant showed significantly lower activity than wild type (Fig 4A). O-glucosyltransferase activity was dependent on the concentrations of EGF repeat and UDP-glucose (acceptor and donor substrate, respectively). POGLUT1 ${ }^{\text {D233E }}$ also showed lower activity than wild type toward five different single EGF repeats from mouse Notch1 (Fig 4B), suggesting that the mutation affects $O$-glucosylation of all EGF repeats containing the $O$-glucose consensus sequence (CXSX(P/A)C) (Rana et al, 2011). Prior work has shown that POGLUT1 also has protein $O$-xylosyltransferase activity toward certain EGF repeats with a diserine motif within the O-glucose consensus sequence (e.g. EGF16 of mouse Notch2) (Takeuchi et al, 2011). POGLUT1 ${ }^{\mathrm{D} 233 \mathrm{E}}$ mutant also showed lower
$O$-xylosyltransferase activity than wild type (Appendix Fig S7B). To examine whether POGLUT1 ${ }^{\text {D233E }}$ retains any enzymatic activity, we performed overnight incubation and then analyzed the products by reverse-phase HPLC and mass spectrometry. Both wild type and the D233E mutant added a single glucose or xylose to the hFIX-EGF repeat (Fig 4C) or EGF16 from mouse Notch2 (Appendix Fig S7C), indicating that POGLUT1 ${ }^{\text {D233E }}$ has residual enzymatic activity.

\section{Analysis of Notch signaling pathway and satellite cells in D233E patients}

Mutations in the Drosophila gene rumi, which encodes the flyPOGLUT1, result in a temperature-sensitive loss of Notch signaling (Acar et al, 2008). Moreover, Poglut1 knockdown in mouse myoblast $\mathrm{C} 2 \mathrm{C} 12$ cells results in a significant decrease in $O$-glucosyltransferase activity and impaired Notch signaling (FernandezValdivia et al, 2011). Accordingly, we examined the expression of activated Notch and the levels of Notch downstream targets in patient muscles. Decreases in Notch1-intracellular domain expression and in HES1 mRNA levels in skeletal muscle were detected compared with controls, which support that D233E affects Notch activity (Fig 5A and B). Notch signaling is critical in normal postnatal myogenesis by regulating SC maintenance (Bjornson et al, 2012), at least in part by directly inducing the expression of Pax7, which encodes a transcription factor critical for the maintenance of adult SC (Olguin \& Olwin, 2004). Indeed, treating proliferating C2C12 cells with the $\gamma$-secretase inhibitor DAPT, which blocks Notch signaling, significantly reduced the mRNA levels of Pax7 (Appendix Fig S8). Likewise, we found a decrease in PAX7 mRNA and fewer $\mathrm{PAX7}^{+}$cells in the muscle of patients compared with controls (Fig 5C and D). Together, these observations suggest that the D233E mutation affects the self-renewal of SC by reducing Notch signaling, likely due to defective $O$-glucosylation.

We analyzed the same parameters in patients suffering from other muscular dystrophies, and also in patients suffering from a secondary dystroglycanopathy, whose muscle biopsies showed mild-to-moderate dystrophic changes, similar to our patients. Both groups show significantly higher levels of activated Notch and higher numbers of $\mathrm{PAX}^{+}$cells compared with D233E patients (Appendix Fig S9A-H). These data support the notion that the observed decrease in Notch1 signaling and PAX7 ${ }^{+}$cells in D233E patients is not secondary to $\alpha$-dystroglycan hypoglycosylation.

\section{In vivo functional study of POGLUT1 ${ }^{\text {D233E }}$}

To investigate the in vivo effects of the D233E mutation on POGLUT1 function in muscle progenitors, we performed crossspecies rescue experiments in the context of adult myogenesis in Drosophila, a process regulated by Notch signaling (Gildor et al, 2012). In these experiments, we used the rumi ${ }^{79}$ allele, which harbors a missense mutation that abolishes fly-POGLUT1 enzymatic activity but does not affect its expression (Acar et al, 2008). When raised at $18^{\circ} \mathrm{C}$, rumi ${ }^{79 / 79}$ animals exhibited normal indirect flight muscle development and a large number of myoblasts expressing the transcription factor Twist, which is a downstream target of Notch activation (Fig 6A-B"). However, when rumi ${ }^{79 / 79}$ animals were raised at 25 and $30^{\circ} \mathrm{C}$ during the early pupal stage, when these muscles form, muscle development was dramatically impaired and 
A

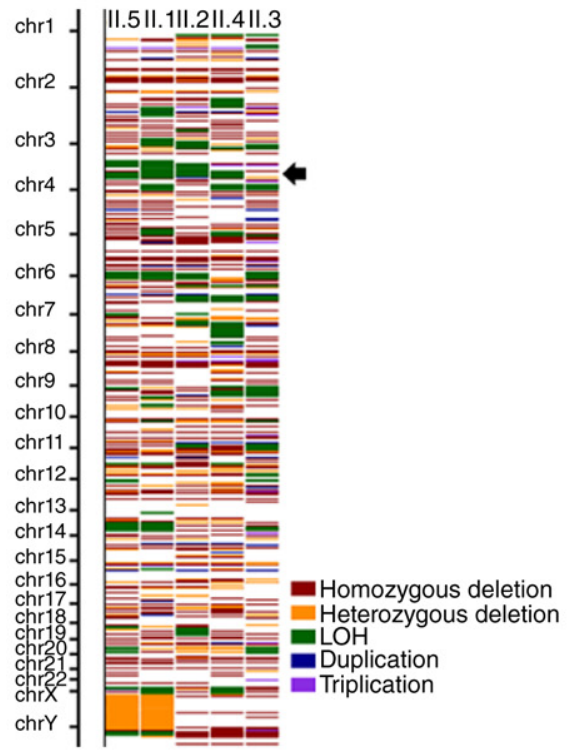

B

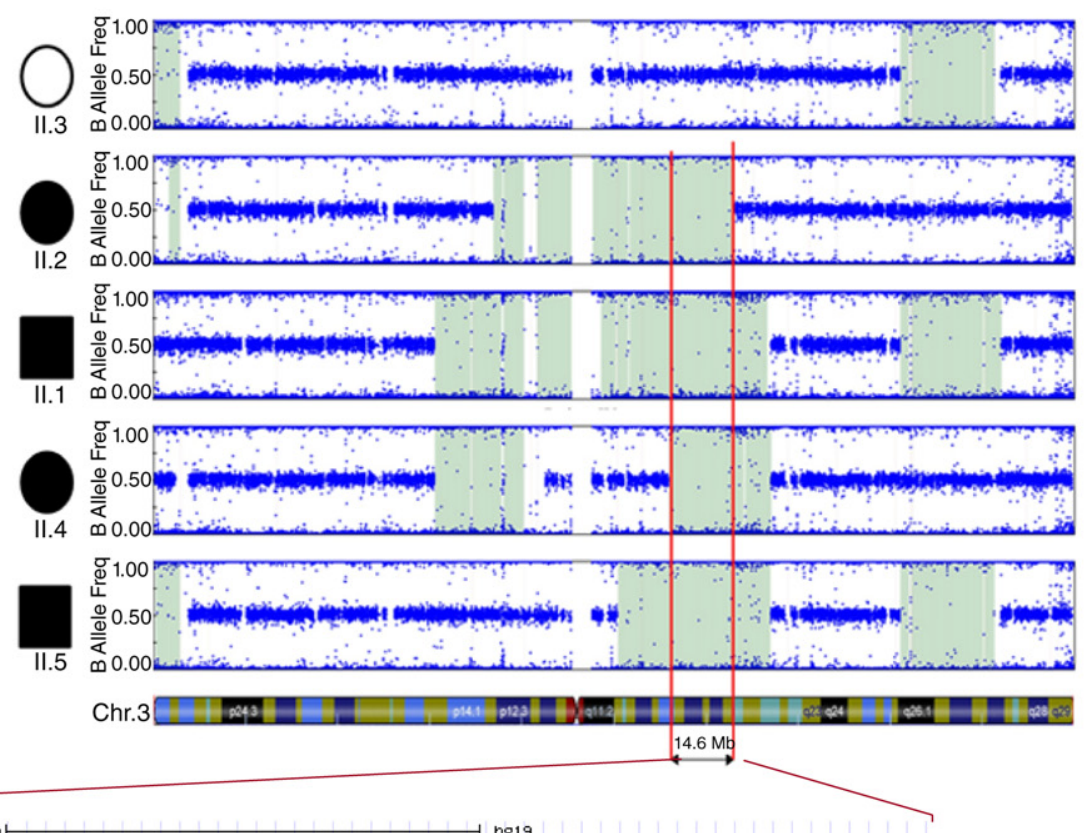

C

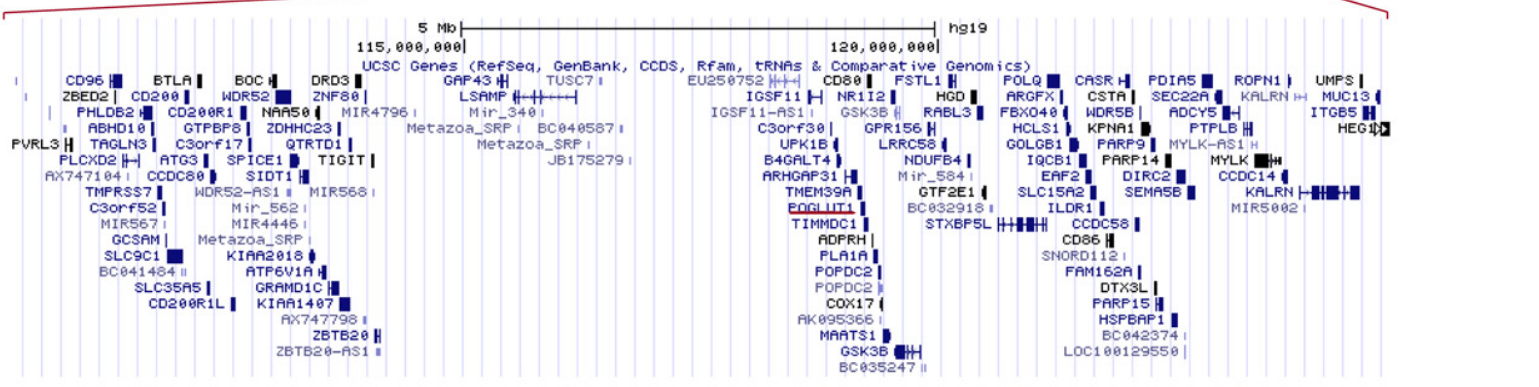

D

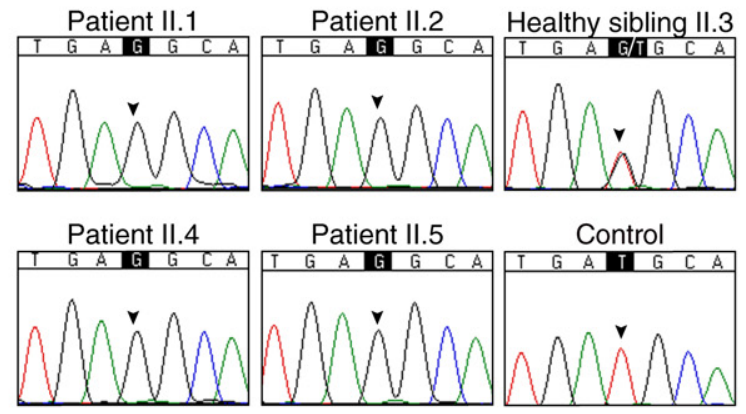

E

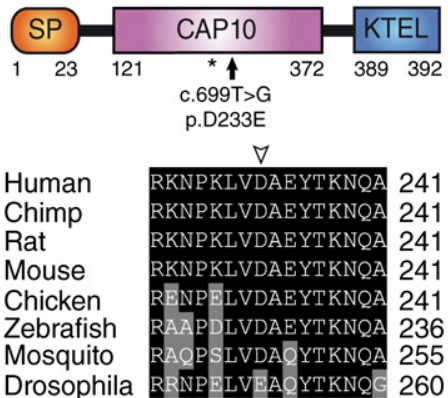

Figure 3. POGLUT1 D233E mutation in patients.

A Genomewide mapping through high-density genotyping. The structural genomic variation and extended regions of homozygosity (loss of heterozygosity [LOH]) over the entire genome is shown for individuals II.1 to II.5. The homozygosity segment shared by the four affected siblings in chromosome 3 is indicated with an arrow.

B This region is represented in more detail, where extended regions of homozygous genotypes in contiguous biallelic polymorphisms are highlighted with a green background.

C The 14.6 megabase region of homozygosity that is shared by all affected siblings is highlighted by vertical red lines in (B), and genes within this genomic region are shown. Note that POGLUT1 gene is underlined.

D Chromatograms reveal a homozygous T-to-G substitution (black boxes and arrowheads) in the four affected family members, while a heterozygous $\mathrm{C} / \mathrm{T}$ in the healthy sibling.

E Schematic structure of the human POGLUT1 protein, which contains a signal peptide (SP), a CAP10 domain, and a Lys-Asp-Glu-Leu (KDEL)-like endoplasmic reticulum retention signal. D233E is located in the CAP10 domain (arrow), close to the predicted ERD catalytic motif (asterisk). Alignment of amino acids flanking the mutated aspartic acid from POGLUT1 orthologs indicates evolutionary conservation of D233 in vertebrates (open arrowhead). Black boxes denote similarity of amino acid residues.

the number of Twist ${ }^{+}$myoblasts was severely decreased (Fig 6C- $\mathrm{D}^{\prime \prime}$ ). The severe temperature-sensitive defects observed in indirect flight muscle development in rumi $i^{79 / 79}$ animals indicate that adult myoblast development in flies depends on the enzymatic activity of fly-POGLUT1. Overexpression of wild-type human-POGLUT1-FLAG in muscle progenitors rescued the muscle phenotypes of $r u m i^{79 / 79}$ 

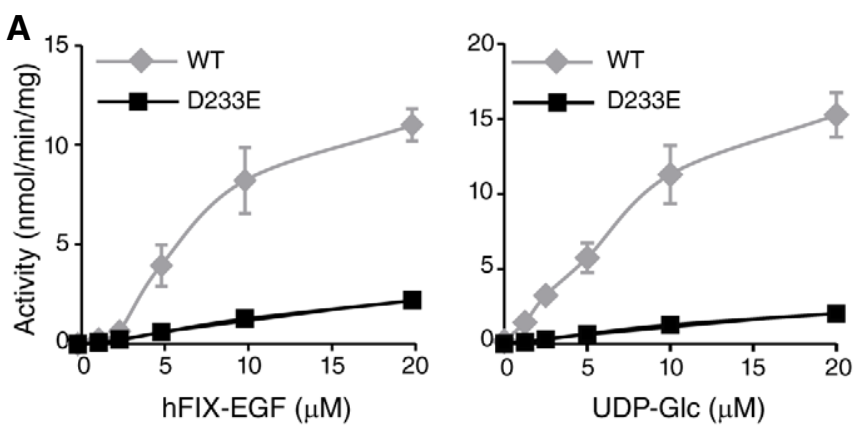

B

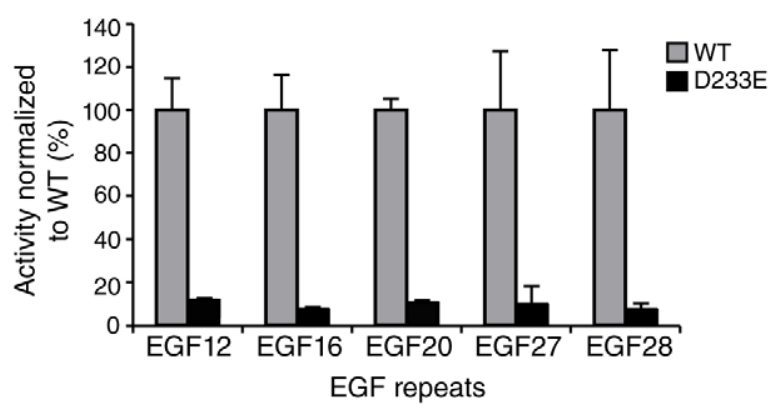

C
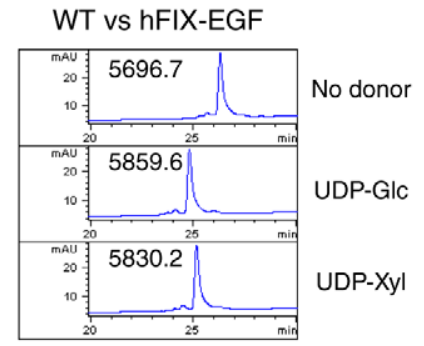

D233E vs hFIX-EGF

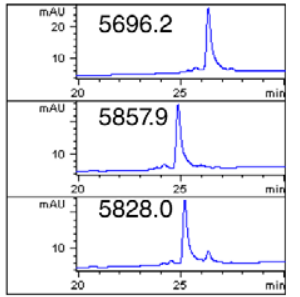

Figure 4. POGLUT1 ${ }^{\mathrm{D} 233 \mathrm{E}}$ has lower enzymatic activity than wild type.

A Protein O-glucosyltransferase activity of wild-type (WT) or D233E mutant POGLUT1 protein toward human factor IX EGF repeat (hFIX-EGF). Wild type shows higher 0 -glucosyltransferase activity than D233E, and this activity is dependent on the concentration of the acceptor substrate hFIX-EGF repeat (left) and on the concentration of donor substrate UDP-glucose (UDP-GIc) (right). Values indicate mean \pm SEM from three independent assays.

B O-Glucosyltransferase activity toward five different single EGF repeats from mouse Notch1. Wild-type POGLUT1 shows higher activity toward all EGF repeats tested than POGLUT1 ${ }^{\text {D233E. }}$. The $y$-axis shows the normalized activity relative to wild-type POGLUT1. EGF repeats were at $10 \mu \mathrm{M}$. Values indicate mean \pm SEM from three independent assays.

C Elution profiles of the POGLUT1 reaction products on reverse-phase HPLC. hFIX-EGF repeat was incubated with wild-type or D233E mutant POGLUT1 and donor substrate, UDP-Glc or UDP-xylose (UDP-Xyl), at $37^{\circ} \mathrm{C}$ overnight. Values on top of the peaks indicate the measured masses. Addition of Glc (162 Da) or xylose (132 Da) to hFIX-EGF (5696.2 Da) by wild-type POGLUT1 caused a shift to an earlier retention time (left). Similarly, the products exhibited a similar shift after incubation with POGLUT1 ${ }^{\text {D233E }}$ (right). These results indicate that POGLUT1 ${ }^{\text {D233E }}$ can add a single glucose or xylose to hFIX-EGF repeats and thus has residual enzymatic activity.

animals (Fig 6E). However, the overexpression of humanPOGLUT1 ${ }^{\text {D233E }}$-FLAG only showed a weak and variable rescue of the muscle morphology and myoblast numbers in $\mathrm{rumi}^{79 / 79}$ animals (Fig $6 \mathrm{~F}-\mathrm{H}$ ), indicating that this mutation significantly decreases the ability of POGLUT1 to promote muscle development and Notch signaling in myoblasts. It is interesting to note that fly-POGLUT1 harbors a glutamic acid (E) at position 252, which is equivalent to D233 in human-POGLUT1 (Fig 3E). Given the robust protein $O$ glucosyltransferase activity of the fly-POGLUT1 and because $35 \%$ of the amino acids in the CAP10 enzymatic domains of human and fly POGLUT1 are divergent (Acar et al, 2008), other amino acid differences between these two proteins are likely to be the reason why the fly protein is fully functional despite harboring the equivalent of the D233E mutation.

\section{Ex vivo analysis of D233E primary myoblasts}

Previous studies have shown that the Notch signaling pathway not only plays an important role in maintaining satellite cell quiescence, but also enhances myoblast proliferation and inhibits myogenic differentiation (Conboy \& Rando, 2002; Bjornson et al, 2012; Mourikis \& Tajbakhsh, 2014).

In order to directly test our hypothesis that the decrease in the POGLUT1 enzymatic activity caused by the D233E mutation disrupts muscle cell function, we explored myogenesis in D233E primary myoblasts from patient muscle biopsies compared with healthy and disease controls (Appendix Fig S10). We quantified the percentage of self-renewing, proliferating, and differentiating cells based on PAX7 and MyoD expression. Quantitative analysis in myoblasts cultured in a growth medium revealed that the percentage of $\mathrm{PAX}^{+} \mathrm{MyoD}^{+}$(proliferating) cells in D233E cells was significantly lower than those in healthy and disease controls (Fig 7A-C). The percentage of $\mathrm{PAX}^{+}$cells and $\mathrm{PAX}^{+} \mathrm{MyoD}^{-}$(self-renewing) cells was significantly decreased in D233E samples (Fig 7D and E), whereas the percentage of $\mathrm{PAX}^{-} \mathrm{MyoD}^{+}$(differentiating) cells was increased (Fig 7F). When myoblasts reached confluence, they were cultured in a differentiation medium. We observed more efficient myoblast fusion into the multinucleated myotubes and higher levels of myogenin expression in D233E cultures compared with controls (Fig 7G-I). The slow proliferation and facilitated differentiation of mutant myogenic cells are in agreement with decreased Notch activity in the muscle of D223E patients.

Due to the scarcity of cells obtained from fresh patient's muscles in culture (likely because of the severe defect in proliferation of D233E myoblasts), we performed immortalization of the primary myoblasts (Mamchaoui et al, 2011), and conversion of skin fibroblasts from patients to myogenic cells by transduction of inducible MyoD (Choi et al, 1990). Thus, we generated two different continuous myogenic cell lines with the D233E mutation, which showed similar phenotypic features, although PAX7 expression started after 1-3 days in culture in the myogenic cell line (Appendix Figs S11 and S12). To examine whether increasing Notch pathway activity can rescue the alterations of the myogenic process observed in D233E immortalized myoblasts during differentiation in culture, we used lentivirus to overexpress NICD1 in these cells and observed a solid reversion of the proliferation defects (Fig 8A and $\mathrm{B}$, Appendix Fig S13A and B). However, we did not observe rescue of the amount of $\mathrm{PAX}^{+}$cells (Fig 8C). It has been described that the effect of Notch on the expression of PAX7 in myogenic cell lines such as $\mathrm{C} 2 \mathrm{C} 12$ is not as potent as in primary myoblasts (Sun et al, 2008). This probably hindered the modification of PAX7 expression during the rescue experiment. In addition, NICD1 overexpression robustly rescued the facilitated differentiation shown by $\mathrm{D} 233 \mathrm{E}$ myoblasts in culture (Fig 8D, Appendix Figs S13C and S14), and this 
A
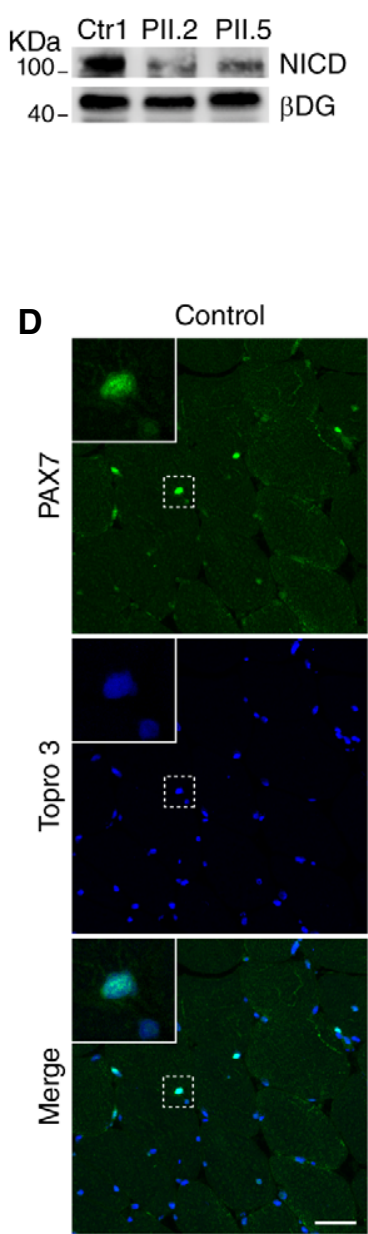

B

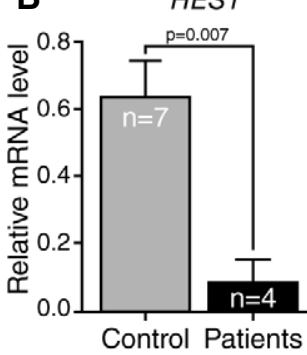

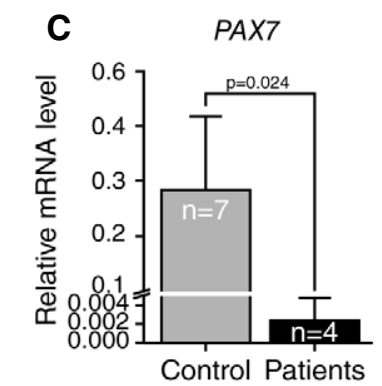

Patient II.5
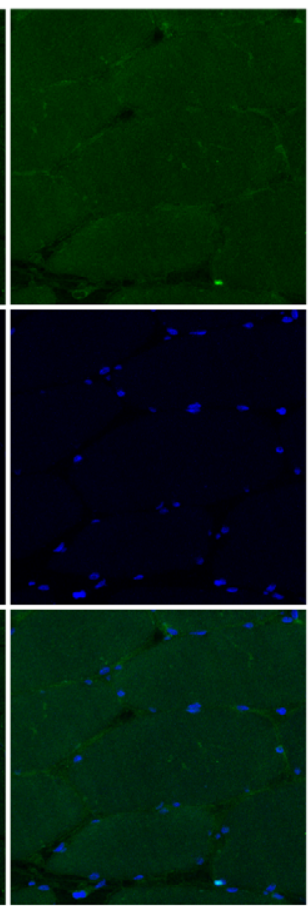

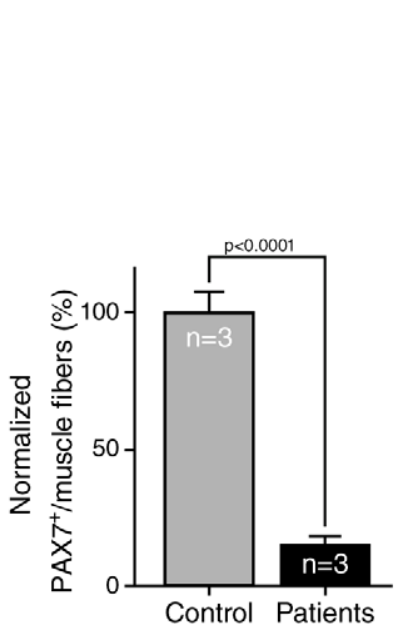

Figure 5. Muscles from D233E patients show decreased Notch signaling and pool of satellite cells.

A Western blot of muscle homogenates shows reduced expression of Notch1 intracellular domain (NICD) in patients.

B, C QRT-PCR on RNA extracted from skeletal muscle shows that expression of HESI (B) and PAX7 (C) was significantly lower in D233E patients compared with healthy controls. Mean \pm SEM; Student's $t$-test (B) and Mann-Whitney U-test (C).

D PAX7 ${ }^{+}$cells in skeletal muscle sections, demonstrating that satellite cells are less abundant in D233E patients than in controls $(n=3$ muscles with $10-15$ fields analyzed per muscle). Mean \pm SEM; Mann-Whitney $U$-test; scale bar, $50 \mu \mathrm{m}$.

Source data are available online for this figure.

rescue was demonstrated by the fusion index (Fig $8 \mathrm{E}$ ) and expression of myogenin (Fig $8 \mathrm{~F}$ ). These results support the pathogenic role of decreased Notch signaling in this novel muscular dystrophy.

\section{Role of Notch on $\alpha$-dystroglycan glycosylation}

As shown in Fig 2, our patients exhibit a muscle-specific reduction in $\alpha$-dystroglycan glycosylation. The only known protein domain to which POGLUT1 can add carbohydrates is a properly folded EGF repeat containing the $\operatorname{CXSX}(\mathrm{P} / \mathrm{A}) \mathrm{C}$ consensus sequence (Takeuchi et al, 2012). Since $\alpha$-dystroglycan contains no EGF repeats nor CXSX (P/A)C sites, it is unlikely to be a direct target of glycosylation by POGLUT1. A recent study has shown that in transgenic mice and
C2C12 myoblasts, $\boldsymbol{\alpha}$-dystroglycan glycosylation is a progressive process that initiates rapidly after myoblasts are induced to differentiate, and exhibits a regular and gradual transition to a larger glycoforms to reach the normal level after 5 days of differentiation (Goddeeris et al, 2013). In order to evaluate this progressive pattern of $\alpha$-dystroglycan glycosylation, primary immortalized myoblasts were differentiated. After 5 days with differentiation medium, D233E myoblasts showed a lower level and irregular progression profile of $\alpha$-dystroglycan glysosylation compared with controls, which reproduced the same pattern previously demonstrated in C2C12 cells (Goddeeris et al, 2013) (Fig 9A). These observations are compatible with abnormal differentiation dynamics in patient myoblasts. Given the well-established role of Notch signaling in 

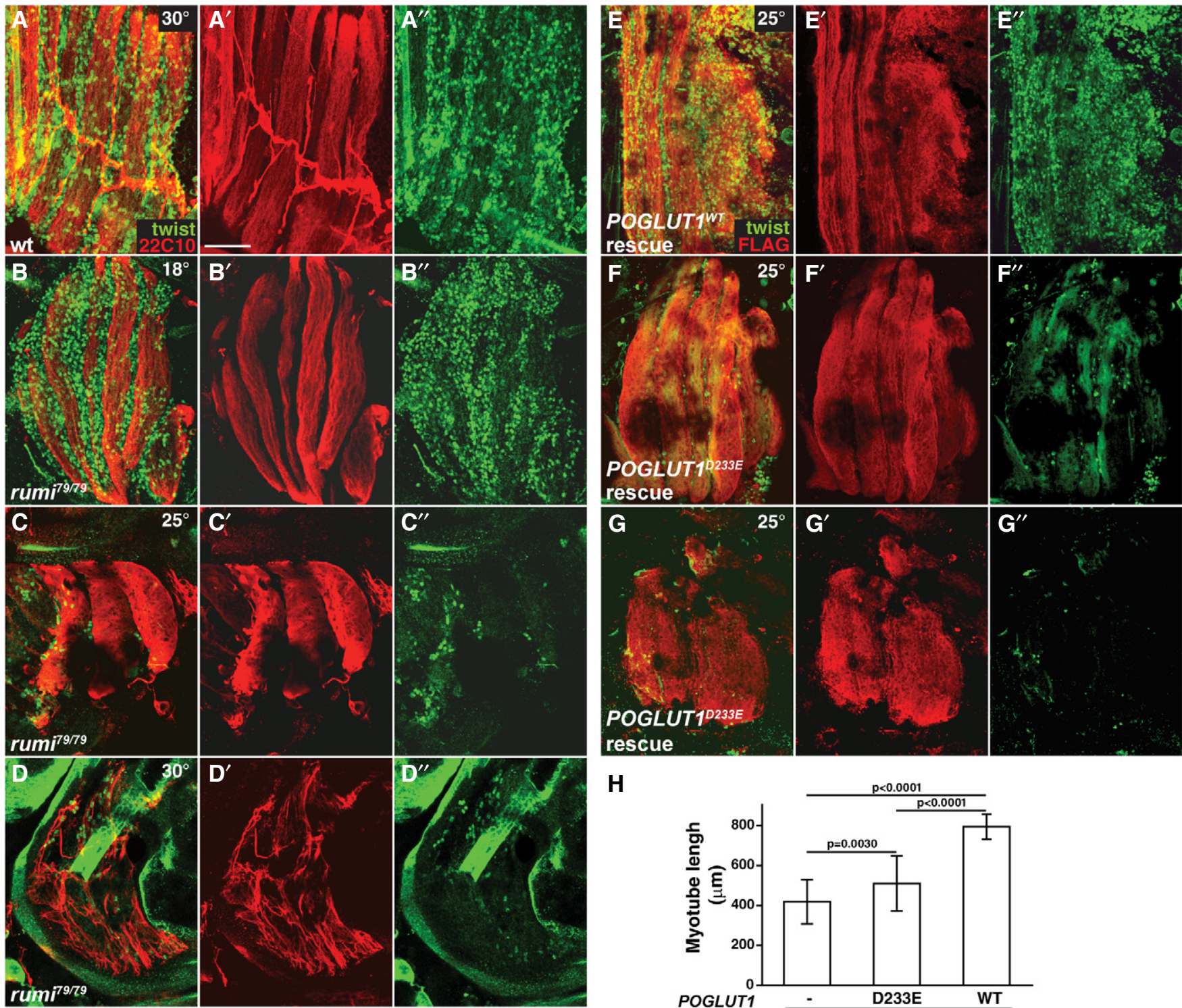

H

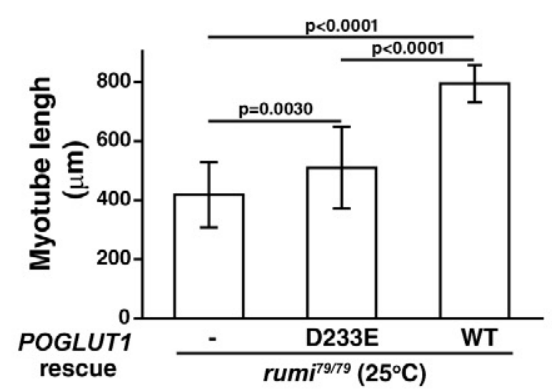

Figure 6. Loss of Rumi results in temperature-sensitive Drosophila muscle defects and is only weakly rescued by POGLUT1 ${ }^{\text {D233E }}$ expression.

A-A" Staining of Drosophila indirect flight muscles at 25\% pupal development with $22 \mathrm{C} 10$ antibody (myotube marker) and anti-Twist antibody (myoblast marker) indicates that in wild-type animals, indirect flight muscles are formed from six myotubes, and a large number of Twist ${ }^{+}$myoblasts are present.

B-B" rumi $i^{7 / 79}$ mutants raised at $18^{\circ} \mathrm{C}$ show no obvious defects in myoblast number or myotube morphology.

C-D" rumi ${ }^{7 / 79}$ mutants exhibit decreased number of myoblasts at $25-30^{\circ} \mathrm{C}$ and either an incomplete set of short myotubes when raised at $25^{\circ} \mathrm{C}$ or aberrant morphology of myotubes when raised at $30^{\circ} \mathrm{C}$.

E-E" rumi ${ }^{7 / 79}$ animals overexpressing FLAG-tagged POGLUT1 ${ }^{\mathrm{WT}}$ in the muscle compartment using Mef2-CAL4 and raised at $25^{\circ} \mathrm{C}$ during the pupal stage show a rescue of the rumi $i^{79 / 79}$ muscle phenotype.

F-G" Overexpression of FLAG-tagged POGLUT1 ${ }^{\text {D233E }}$ only partially rescues the rumi $i^{79 / 79}$ muscle phenotype, with some variation in the myotube length and the number of restored Twist ${ }^{+}$cells (compare G and F). Note that the number of Twist ${ }^{+}$cells restored by POGLUT1 ${ }^{\text {D233E }}$ is much smaller than that restored by POGLUT1 ${ }^{\text {WT }}$.

H Quantification of myotube lengths in rumi ${ }^{79 / 79}$ mutants with or without POGLUT1 expression indicates a weak rescue of the phenotypes by POGLUT1 ${ }^{\text {D233E }}$ compared with POGLUT1 ${ }^{\text {WT }}$. The differences in myotube lengths are statistically significant. For rumi ${ }^{79 / 79}$ rescue (left bar), 5 animals were used and a total of 23 myotubes were measured; for D233E rescue, 11 animals and 54 myotubes; for WT rescue, 5 animals and 22 myotubes. Mean \pm SD is shown; one-way ANOVA with Bonferroni's multiple comparisons test.

Data information: Scale bar in $\left(A^{\prime}\right)$ is $50 \mu \mathrm{m}$ and applies to $\left(A-C^{\prime \prime}\right)$.

Source data are available online for this figure.

muscle differentiation, we hypothesized that decreased Notch signaling in D233E patients' myoblasts might contribute to altered $\alpha$-dystroglycan glycosylation. We first attempted to test this hypothesis by asking whether NICD overexpression can restore $\alpha$-dystroglycan glycosylation in patient myoblasts. However, given the strong inhibition of differentiation upon NICD overexpression, 
A
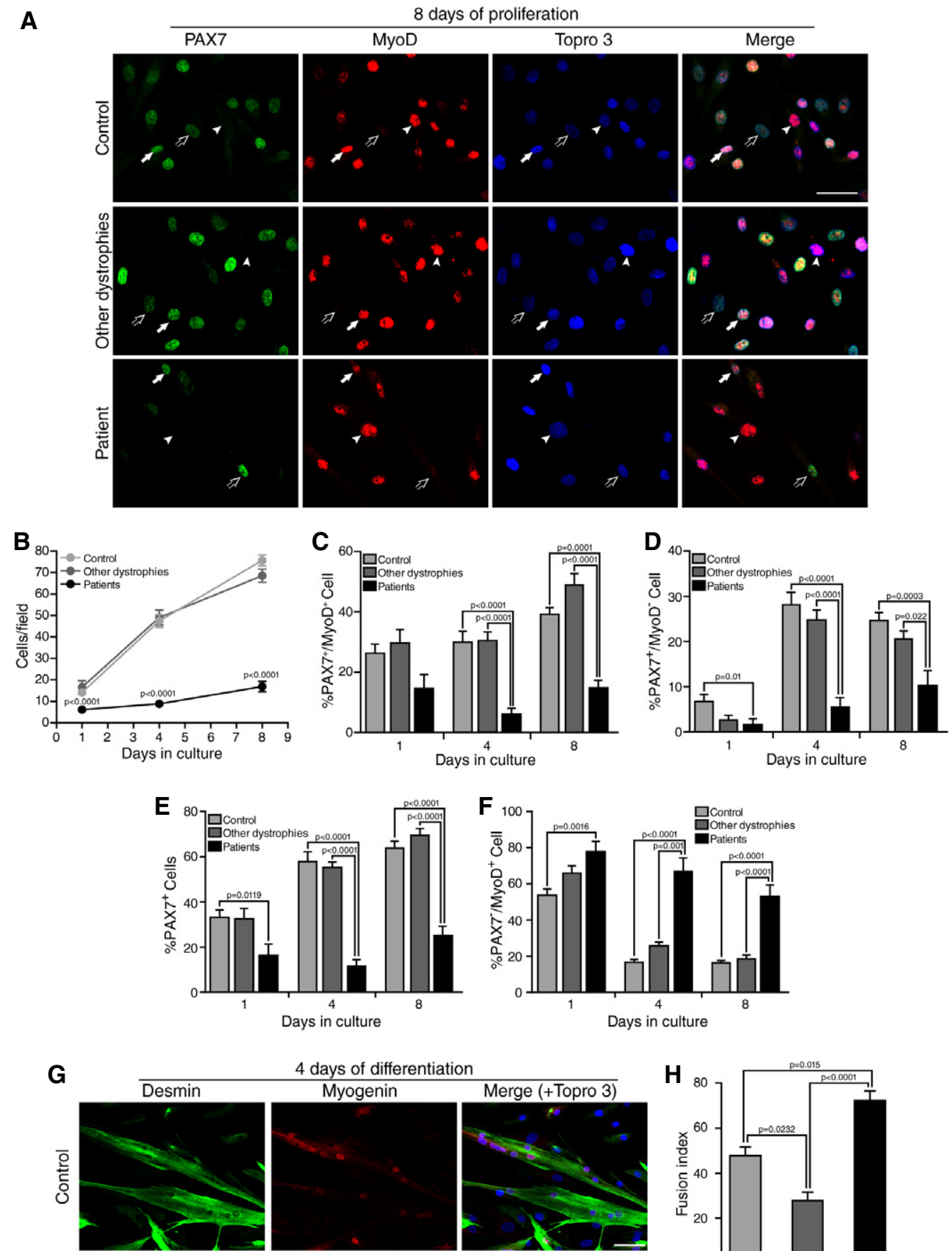

4 days of differentiation
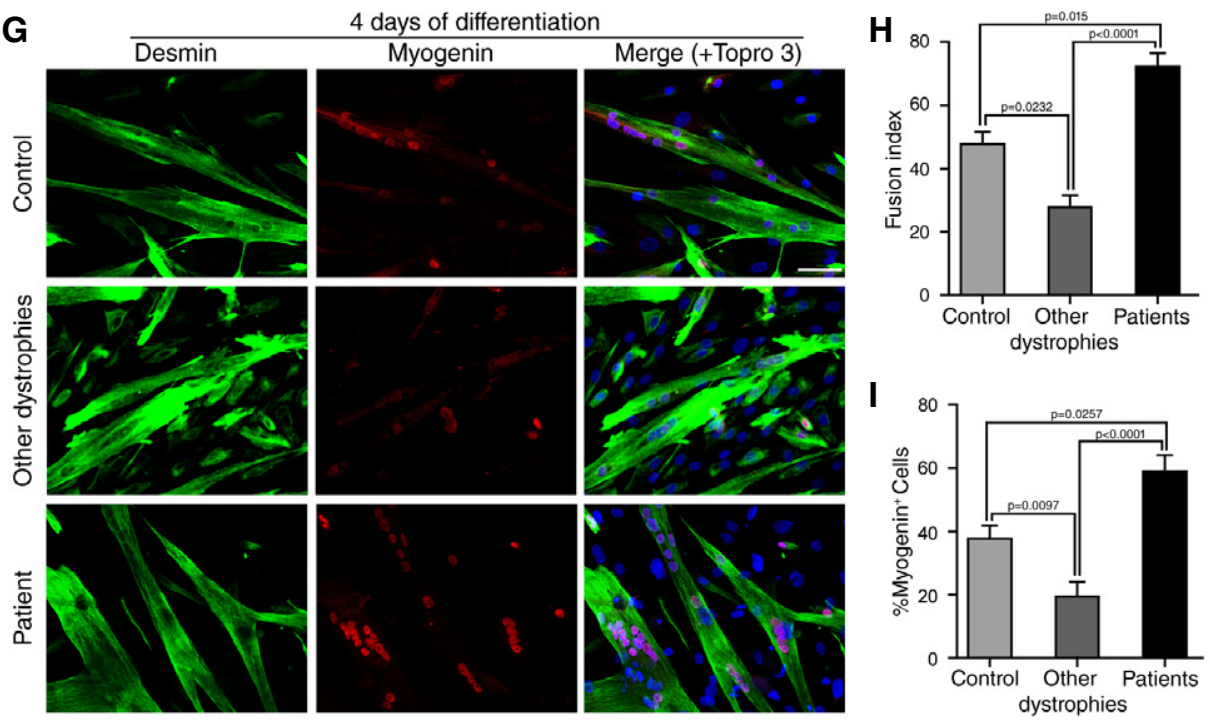

Figure 7. 
Figure 7. D233E primary myoblasts exhibit decreased proliferation and increased differentiation.

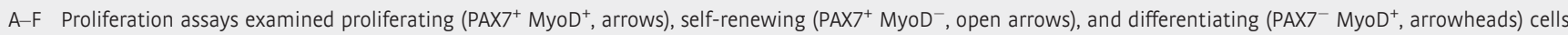
from D233E patients $(n=2)$, healthy controls $(n=3)$, and disease controls $(n=3)$ at 1,4 , and 8 days growing in proliferation medium. Panel (A) shows representative images from day 8 . The number of patient myoblasts is lower than that of controls at all three time points, indicating a slower proliferation rate in patient myoblasts (B), and accordingly, the percentage of proliferating cells (C) is smaller in the patient. (D) The percentage of self-renewing cells is small in patient's culture, reflecting a poor capacity for maintaining the pool of quiescent SCs. In addition, the percentage of PAX7 ${ }^{+}$cells (E) is smaller in patient's culture, similar to what was found in adult D233E muscle (shown in Fig 5D). (F) The percentage of differentiating cells is higher in the patient. We examined proliferation capturing 7-12 randomly fields per day and condition (mean \pm SEM is shown; Kruskal-Wallis with Dunn's multiple comparisons test; scale bar, $50 \mu \mathrm{m}$ ).

G-I When myoblasts started to be confluent, the proliferation medium was replaced with differentiation medium, and the myoblasts started to fuse yielding myotubes, which were analyzed after 4 days of differentiation. $(H)$ The fusion index, which measures the percentage of nuclei in myotubes with respect to the total number of nuclei in myogenic cells, was higher in D233E cultures than in controls. (I) Besides, myogenin showed increased expression in D233E myoblast cultures compared with controls. The data support that differentiation process is facilitated in patient's muscle. Data were collected from 3 to 10 randomly chosen areas per condition (mean \pm SEM is shown; Kruskal-Wallis with Dunn's multiple comparisons test (H) and one-way ANOVA with Bonferroni's multiple comparisons test (I); scale bar, $50 \mu \mathrm{m})$.

Source data are available online for this figure.

we were unable to do this, as in both cases (rescued and not rescued patient's myoblasts) $\alpha$-dystroglycan glycosylation was immature (Fig 9B). Next, we examined whether inhibition of Notch signaling leads to altered pattern of $\alpha$-dystroglycan glycosylation during differentiation. To this end, we differentiated $\mathrm{C} 2 \mathrm{C} 12$ cells with medium containing DAPT or LY3039478 to decrease Notch signaling. The cells showed a reduced expression of glycosylated $\alpha$-dystroglycan at all differentiation days (Fig 9C-F). These results support the notion that $\alpha$-dystroglycan hypoglycosylation is the consequence of Notch inactivation, produced by the reduced activity of POGLUT1 in the case of our patients.

\section{Discussion}

Transgenic mice in which the pan-Notch inhibitor dnMAML1 is specifically expressed in muscle SC were recently shown to display reduced PAX7 expression in SC cells and muscular dystrophic features even without experimentally induced injury (Lin et al, 2013). This study highlighted the role of Notch signaling and SCs in repairing the muscle damage caused by regular activity in a mammal, suggesting that a primary defect in SC maintenance caused by decreased Notch signaling can in principle result in muscular dystrophy. However, direct evidence from human patients was lacking to support this notion. Here, we describe a family in which a recessive D233E mutation severely impairs the enzymatic activity of POGLUT1, leading to a marked reduction in Notch activation in adult muscle, a decreased pool of $\mathrm{PAX}^{+} \mathrm{SCs}$, and a partial defect of $\alpha$-dystroglycan functional glycosylation. The findings in this family sharply contrast with those in other forms of age-matched limb-girdle muscular dystrophy, including secondary dystroglycanopathies, which show normal levels of HES1 and PAX7 transcripts in adult muscles (Appendix Fig S9).

Although patients' muscles display reduced glycosylation of $\alpha$-dystroglycan, several findings are inconsistent with a secondary dystroglycanopathy. First, the patients do not have elevated creatine kinase and muscle biopsies do not show many degenerative fibers compared with that observed in secondary dystroglycanopathies (Cirak et al, 2013). Second, the D233E muscles exhibit reduced $\alpha$-dystroglycan laminin-binding activity but show normal agrinbinding and compact basement membrane ultrastructure. Although it is known that LARGE-dependent glycosylation of $\alpha$-dystroglycan is required for the binding of both laminin and agrin, the precise glycan structures recognized by these ligands have not been determined. Agrin, but not laminin, showed normal binding to $\alpha$-dystroglycan in patients' muscle, suggesting that these ligands may recognize slightly different glycan structures on $\alpha$-dystroglycan. Third, $\alpha$-dystroglycan expression and laminin binding are normal in D233E skin fibroblasts. In contrast, other forms of dystroglycanopathy usually exhibit markedly diminished or no binding for both laminin and agrin in muscle, together with defective muscular basement membrane compaction (Michele et al, 2002; Hara et al, 2011; Willer et al, 2012; Goddeeris et al, 2013). Moreover, the published results show that secondary dystroglycanopathies caused by mutations in known genes result in reduced functional $\alpha$-dystroglycan glycosylation in patient fibroblasts, and in the majority of patients, the level of $\alpha$-dystroglycan glycosylation is comparable in fibroblasts and skeletal muscle from the same patient (Carss et al, 2013). These observations further support the notion that, although decreased laminin binding in muscle is likely to contribute to pathology in our

Figure 8. Rescue experiments on immortalized primary myoblasts by lentiviral infection.

A-C We overexpressed NICD in immortalized primary myoblasts to rescue the features displayed by immortalized D233E myoblasts. (B) We analyzed the proliferation at 1,3 , and 5 days, and proliferation rate in patient-LV-NICD-GFP reached the level observed in control-LV-GFP, which were higher than in patient-LV-GFP. (C) However, the percentage of PAX7 $7^{+}$cells in patient-LV-NICD-GFP showed the same reduced value as in patient-LV-GFP, which were significantly lower than in control-LV-GFP. The majority of control myoblasts were proliferating cells (PAX7 ${ }^{+}$MyoD ${ }^{+}$, arrows), with no quiescent cells and few differentiating cells (PAX7 $M_{y o D^{+}}$, arrowheads) detected. Data were collected from $n=2$ independent assays (5-8 images per condition). Mean \pm SEM is shown; one-way ANOVA with Tukey multiple comparisons test (B) and Kruskal-Wallis with Dunn's multiple comparisons test (C); scale bar, $50 \mu \mathrm{m}$.

D-F As previously described in patient's primary myoblasts, patient-LV-GFP myoblasts showed a striking facilitated differentiation compared with control-LV-GFP myoblasts. This phenotype fully disappeared in patient-LV-GFP-NICD myoblasts, as quantification of the fusion index (E), and myogenin expression (F) demonstrated. The data support a pathogenic role for Notch inhibition on the altered differentiation in our patients. Data were collected from $n=3$ independent assays (13-17 images per condition). Mean \pm SEM is shown; Kruskal-Wallis with Dunn's multiple comparisons test; scale bar, $50 \mu$ m.

Source data are available online for this figure. 


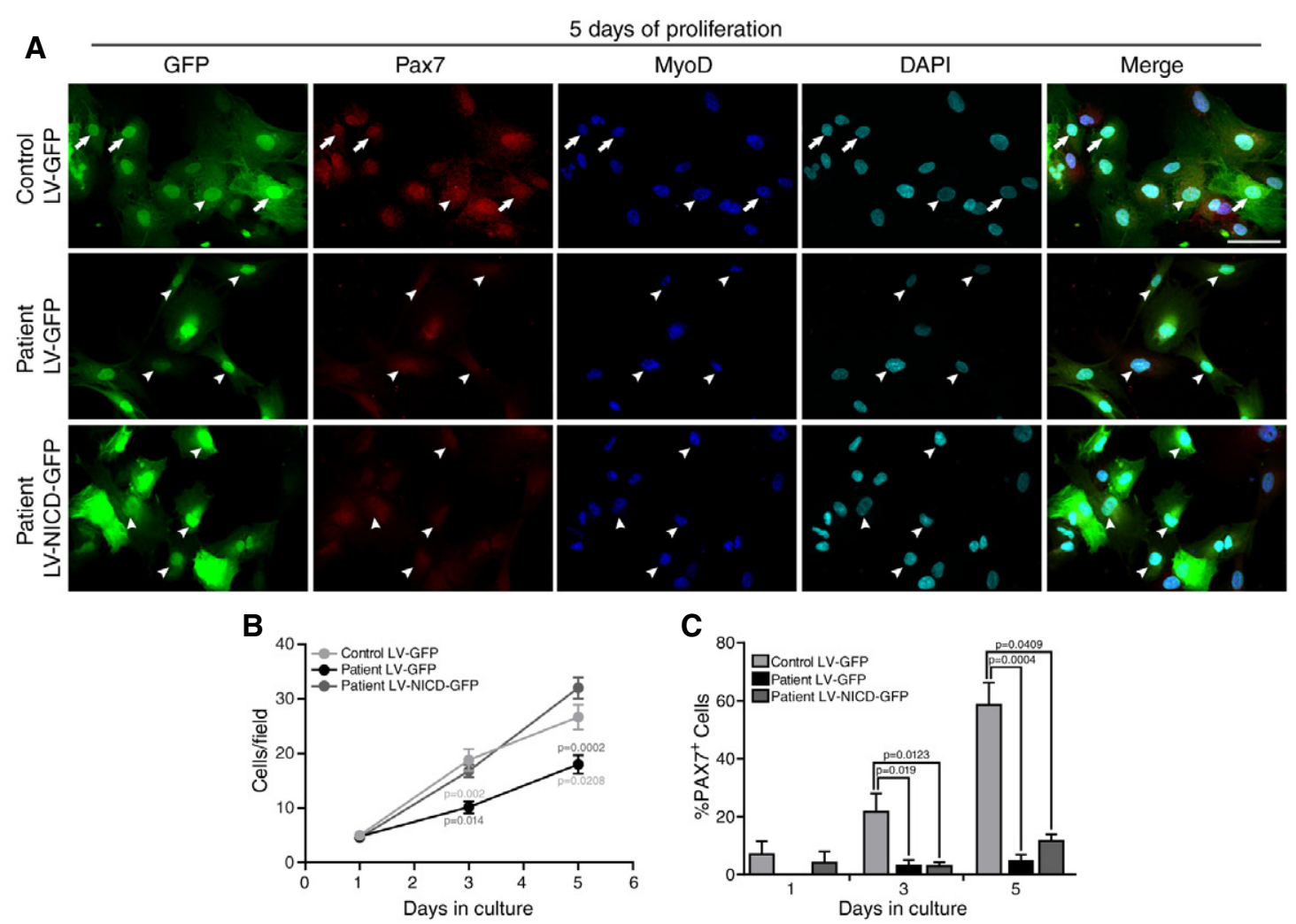

5 days of proliferation
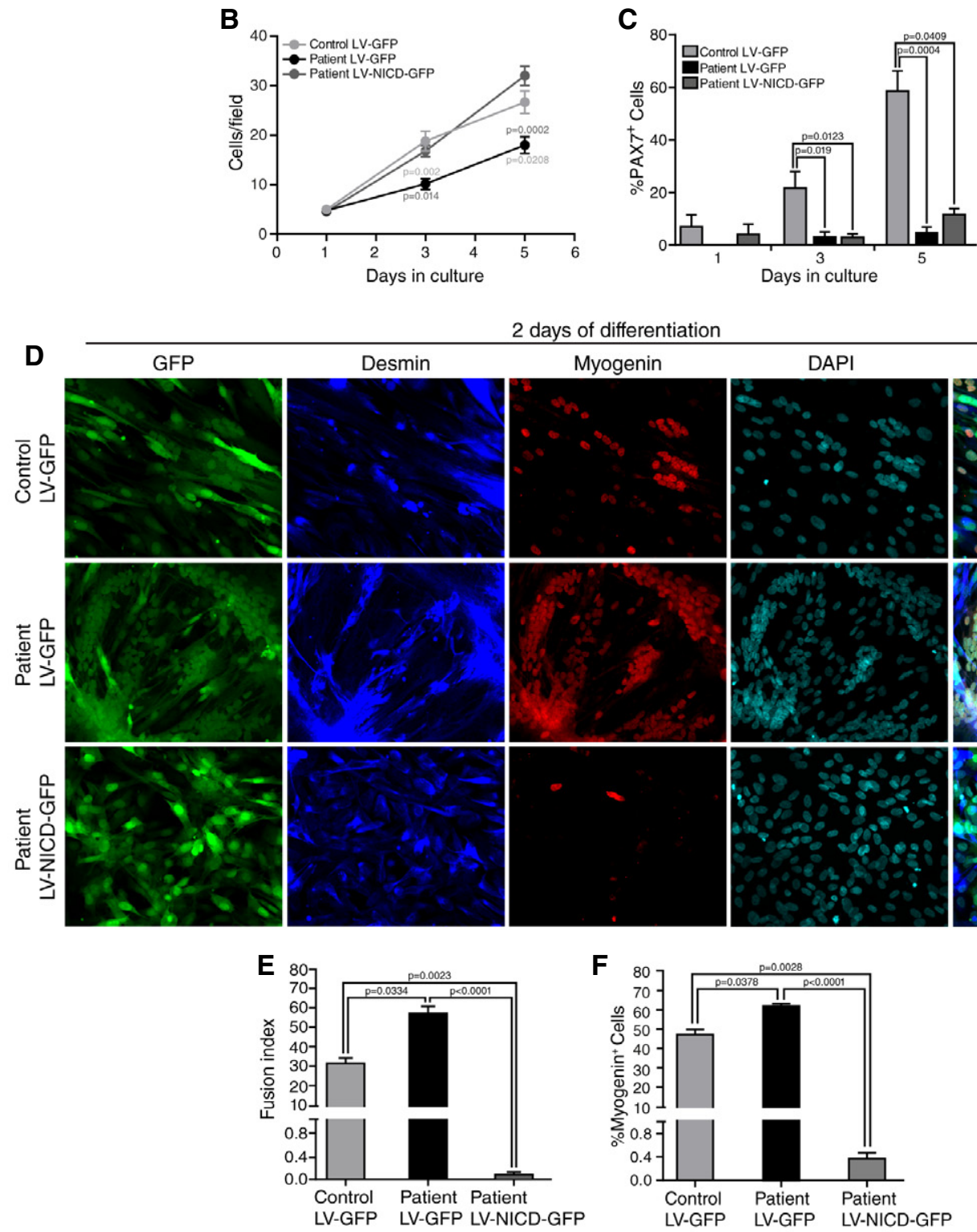

2 days of differentiation

Myogenin
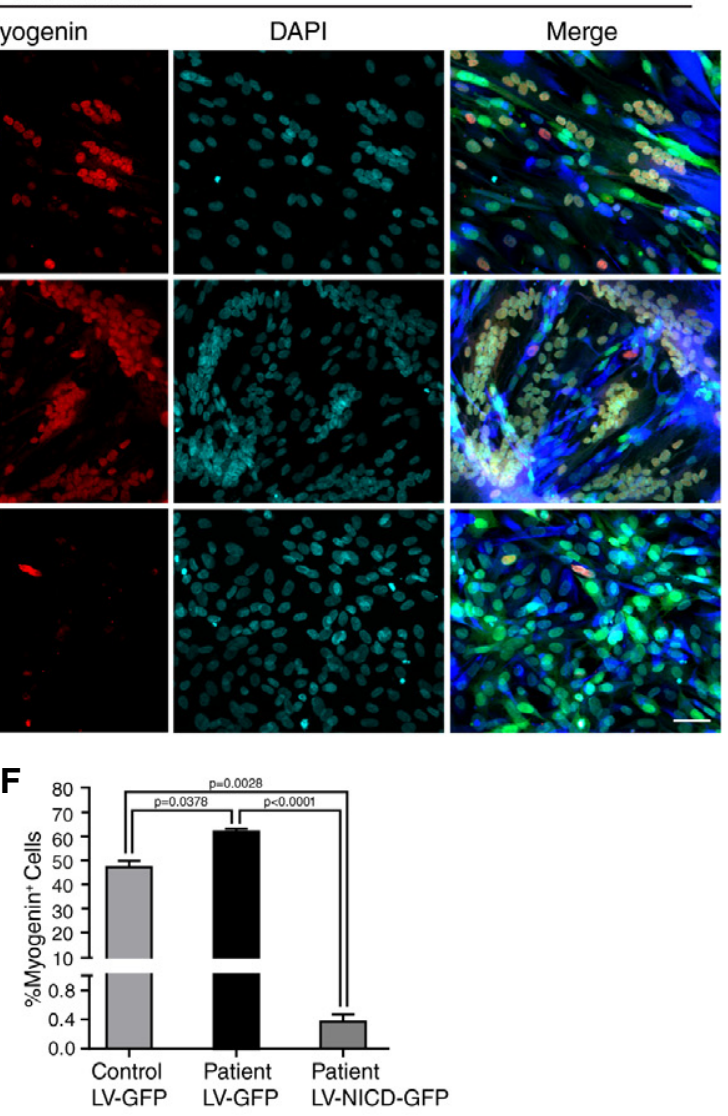

Figure 8. 

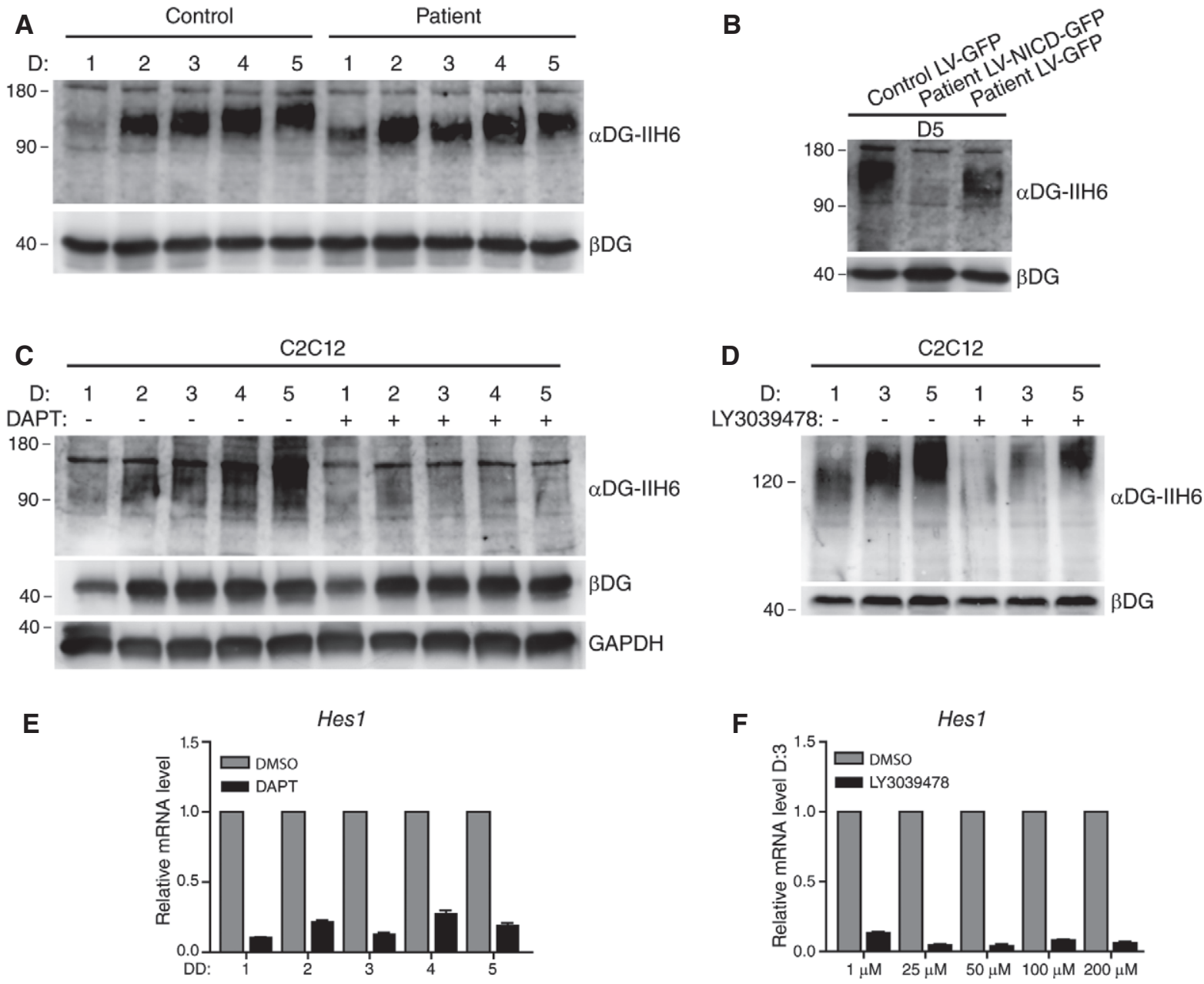

Figure 9. Effects of decreased Notch signaling on $\alpha$-dystroglycan glycosylation during differentiation.

A Immortalized primary myoblasts from a healthy control showed a progressive pattern of $\alpha$-dystroglycan glycosylation during 5 days of differentiation (D0-D5), as has been previously described by Goddeeris et al (2013). Immortalized primary myoblasts from patients displayed an irregular pattern of glycosylation, with a final level of glycosylated $\alpha$-dystroglycan that was lower than control.

B $\quad \alpha$-dystroglycan glycosylation of infected immortalized myoblasts from patient-LV-NICD-GFP at differentiation day 5 (D5) showed a striking reduction, in line with the strong inhibition of myotubes formation and differentiation induced by NICD overexpression. $\alpha$-dystroglycan in myoblasts from patient-LV-GFP showed a reduction in glycosylation compared with control-LV-GFP at D5, similar to the results obtained in the adult muscle from patients (Fig 2).

C, D C2C12 myogenic cells showed a reduced level of glycosylated $\alpha$-dystroglycan when the Notch signaling inhibitor DAPT or LY3039478 was added to the differentiation medium. This indicates that reducing Notch signaling can affect $\alpha$-dystroglycan glycosylation in wild-type C2C12 myoblasts cells, which do not harbor any known mutations in $\alpha$-dystroglycan glycosyltransferases or Poglut1.

E, F qRT-PCR experiments show that upon DAPT or LY3039478 treatment, a remarkable decrease in Hes I mRNA is induced in C2C12 cells during differentiation. Three replicates per condition were analyzed; mean \pm SEM is shown.

Source data are available online for this figure.

patients, $\boldsymbol{\alpha}$-dystroglycan hypoglycosylation might not be the disease mechanism caused by the D233E mutation, or at least the primary mechanism.

Strikingly, our data suggest that the D233E mutation causes defects in SC proliferation and differentiation. The amount number of SCs in our patients is significantly reduced even in very early stages when the muscle is barely affected, compared with healthy and disease controls. Alterations in SC proliferation have been described in the Large ${ }^{\text {myd }}$ mouse dystroglycanopathy model and are thought to contribute to myodegeneration in these animals (Ross et al, 2012). In this model, SCs are significantly increased at early stages and have reduced proliferation when cultured on freshly isolated single fibers; however, proliferation is restored in culture when SCs are removed from the fibers, indicating that rather than intrinsic defects in myoblasts, compositional changes in the extracellular matrix due to hypoglycosylated $\alpha$-dystroglycan are responsible for the proliferative alteration. In contrast, myoblasts from our patients displayed reduced proliferation and self-renewal capacity in culture when removed from their fiber niche, supporting an intrinsic defect in D233E myoblasts independent of $\alpha$-dystroglycan hypoglycosylation. Moreover, it has been recently demonstrated in dystroglycanopathy mice models that functional glycosylation of 
$\alpha$-dystroglycan does not play a significant role in myogenic cell proliferation and differentiation (Awano et al, 2015). Our results indicate that the D233E mutation results in deficient Notch signaling in patient muscle and an impairment in the regulation of myoblast differentiation and that expression of NICD rescues this phenotype. Our results strongly suggest that impaired myogenesis mediated by decreased Notch signaling is a causal mechanism of muscular dystrophy in our patients (Fig 8). Although we expected that the reduction in $\mathrm{PAX7}^{+}$cells in our patients would be a direct effect of Notch inhibition, the lack of rescue of $\mathrm{PAX}^{+}$cells by NICD overexpression suggests something more complicated. The effect of Notch on PAX7 expression has been reported to be weaker in myogenic cell lines than in primary myoblasts, and this could be affecting the rescue of $\mathrm{PAX}^{+}$cells in our system (Sun et al, 2008). Nevertheless, our data consistently demonstrated a decreased number of $\mathrm{PAX7}^{+}$ cells in D233E patients, compared with healthy and disease controls, including secondary dystroglycanopathies. This result together with a previous report showing that the blockade of Notch signaling in muscle SCs of a mouse model causes muscular dystrophy and impairs muscle regeneration (Lin et al, 2013) supports our hypothesis that impaired regulation of muscle SC homeostasis plays a pathogenic role in this new muscle dystrophy. Recent findings demonstrate that the overexpression of Jagged1, a major ligand of the Notch signaling pathway, ameliorates the dystrophic phenotype in the golden retriever muscular dystrophic dogs, which is an excellent model to the human dystrophynopathy, indicating that the Notch pathway can be considered a new therapeutic target in muscular dystrophies (Vieira et al, 2015).

POGLUT1 specifically adds $O$-glucose or $O$-xylose to properly folded EGF repeats containing the $\mathrm{CXSX}(\mathrm{P} / \mathrm{A}) \mathrm{C}$ consensus sequence (Rana et al, 2011; Takeuchi et al, 2011, 2012) and is among the glycosyltransferases that directly add sugar molecules to an amino acid, not to other sugars. Therefore, since $\alpha$-dystroglycan lacks EGF repeats and the $\operatorname{CXSX}(\mathrm{P} / \mathrm{A}) \mathrm{C}$ consensus sequence, POGLUT1 is highly unlikely to directly add carbohydrates to $\alpha$-dystroglycan core protein or to extend the carbohydrate chains added to $\alpha$-dystroglycan by other glycosyltransferases. This is supported by our observation that glycosylation of $\alpha$-dystroglycan from D233E skin fibroblasts is normal. These data strongly argue that POGLUT1 does not participate in the biosynthesis of the $O$-mannose glycans on $\alpha$-dystroglycan. Therefore, unlike POMT1/2 and LARGE which add carbohydrates to $\alpha$-dystroglycan to regulate muscle maintenance, POGLUT1 most likely glycosylates other target proteins to maintain muscle integrity. This notion is supported by our fly experiments: Loss of POGLUT1 enzymatic activity in Drosophila also causes a severe decrease in the number of Twist $^{+}$myoblasts and muscle development, which can be fully rescued by wild-type human-POGLUT1 but only weakly so by POGLUT1 ${ }^{\text {D233E }}$. However, Drosophila dystroglycanopathy models, including null alleles of dystroglycan, do not have defects in myoblast migration and myofiber formation (Nakamura et al, 2010), indicating that the muscle phenotypes observed in rumi flies are not caused by alterations in dystroglycan.

Fourteen Drosophila proteins and about 50 mammalian proteins have EGF repeats with a $\operatorname{CXSX}(\mathrm{P} / \mathrm{A}) \mathrm{C}$ consensus sequence and are therefore predicted to be modified by POGLUT1 (Jafar-Nejad et al, 2010; Rana et al, 2011; Haltom et al, 2014). This consensus motif is highly predictive of enzymatic modification, as mass spectrometric analyses have demonstrated that most if not all EGF repeats with a
CXSX(P/A)C motif examined so far are O-glucosylated (Hase et al, 1988; Acar et al, 2008; Fernandez-Valdivia et al, 2011; Rana et al, 2011; Lee et al, 2013; Haltom et al, 2014; Ramkumar et al, 2015; Thakurdas et al, 2016). Agrin, which is implicated in muscle disease, is predicted to have a single POGLUT1 target site. However, we did not observe any defects in agrin's level, molecular size, or its capacity to bind $\alpha$-dystroglycan in our patient muscles, strongly suggesting that impaired agrin glycosylation cannot explain the muscular dystrophy in our patients. Indeed, the addition of $O$-glucose to a target protein by POGLUT1 does not necessarily indicate functional importance. For example, even though Drosophila Crumbs is O-glucosylated, homozygosity for a knock-in allele of the Drosophila crumbs in which all seven Crumbs $O$-glucosylation sites are mutated does not recapitulate crumbs loss-of-function phenotypes during fly development and generates viable and fertile adults (Haltom et al, 2014). Among the confirmed POGLUT1 biochemical targets, Drosophila Notch, mouse Notch1, mouse CRUMBS2, and Drosophila Eyes shut are shown to be functionally regulated by POGLUT1-mediated glycosylation (Acar et al, 2008; Fernandez-Valdivia et al, 2011; Leonardi et al, 2011; Haltom et al, 2014; Ramkumar et al, 2015). Moreover, there is evidence suggesting that mouse JAG1 might also be a biologically relevant target of POGLUT1, although further experiments are required to prove this (Thakurdas et al, 2016). Based on the severe decrease in Notch signaling and Notch1 cleavage in our patients' muscle, the dramatic decrease in the ability of POGLUT1 ${ }^{\text {D233E }}$ to glycosylate Notch EGF repeats, and previous observations on the role of POGLUT1 and Notch receptor O-glucosylation in Notch signaling (Acar et al, 2008; Fernandez-Valdivia et al, 2011; Leonardi et al, 2011; Ma et al, 2011; Rana et al, 2011), we propose that the Notch receptors are the key target of POGLUT1 in the muscle. However, we cannot exclude that other POGLUT1 targets might also contribute to the phenotypes observed in our patients.

If POGLUT1 affects a glycosylation pathway unrelated to the $O$ mannose glycans, it raises the question of why $O$-mannose glycosylation on $\alpha$-dystroglycan is impaired in D233E muscle. Our data strongly favor an indirect mechanism for $\alpha$-dystroglycan hypoglycosylation, likely due to altered myogenesis caused by decreased Notch signaling resulting from the D233E mutation. Indeed, a recent report showed that $\alpha$-dystroglycan glycosylation is at a low level during the first stages of muscle regeneration even in wild-type mice and is increased as myogenesis proceeds (Goddeeris et al, 2013). The authors proposed that coordination between myogenic differentiation and increased expression of the glycosyltransferase LARGE results in bulkier $\alpha$-dystroglycan molecules with more extensive LARGE-mediated glycosylation and thereby a more robust ligandbinding capacity during muscle repair (Goddeeris et al, 2013). Accordingly, $\alpha$-dystroglycan hypoglycosylation in our patients can be a consequence of altered SC homeostasis and differentiation, which would impede muscle repair and potentially result in immature LARGE-mediated glycosylation (Fig 9). Previous data show that an adequate balance of Notch activation is needed during myogenesis (Kuang et al, 2007; Sacco et al, 2008), and our results support an additional critical role of Notch signaling in the process of $\alpha$-dystroglycan glycosylation during muscle differentiation. Nonetheless, the muscle-specific $\alpha$-dystroglycan hypoglycosylation could contribute to muscle dystrophy in our patients.

An autosomal-dominant dermatosis has recently been associated with heterozygous mutations in POFUT1 (protein $O$-fucosyltransferase 
1, which adds $O$-fucose to Notch), and POGLUT1 (Li et al, 2013; Basmanav et al, 2014). Drosophila Pofut1 has a chaperone-like function on the Notch protein independent of its $O$-fucosyltransferase activity (Okajima et al, 2005). Therefore, since patients homozygous for D233E have deficient enzymatic activity but normal POGLUT1 expression and no skin abnormalities, this dermatosis could be due to a defect in a non-enzymatic function of POGLUT1. However, genetic background differences could also exist that make these patients more sensitive to a $50 \%$ reduction in POGLUT1 enzymatic activity.

In conclusion, we identified the human POGLUT1 D233E mutation that impairs Notch posttranslational modification and maintenance of muscle stem cells and leads to muscular degeneration and $\alpha$-dystroglycan hypoglycosylation. Our findings demonstrate that D233E mutation in POGLUT1 causes autosomal recessive limb-girdle muscular dystrophy and implicate a primary defect in muscle progenitor cells as a novel pathomechanism for muscular dystrophy. Our patients display an adult-onset pure muscular phenotype, in contrast to the lethal, multisystemic phenotypes observed in animals with complete loss of POGLUT1 (Acar et al, 2008; Fernandez-Valdivia et al, 2011). Thus, the residual $O$-glucosyltransferase activity in our patients is sufficient for other aspects of human development and tissue homeostasis, indicating that myoblasts are particularly sensitive to decreased POGLUT1 activity.

\section{Materials and Methods}

\section{Skeletal muscle histology and ultrastructural study}

Open muscle biopsies were obtained from biceps brachii or quadriceps of the four patients and the healthy sibling in generation II of the family. The muscle was rapidly frozen in liquid nitrogen-chilled isopentane and $7-\mu \mathrm{m}$-thick cryostat sections were stained for standard histological and histochemical techniques including hematoxylin and eosin, Gomori trichrome, nicotinamide adenine dinucleotide dehydrogenase (NADH), and ATPase pH 9.4. Stained sections were evaluated with an Olympus BX41 (Tokyo, Japan) equipped with a ColorView IIIu camera (Olympus).

A piece of muscle from each biopsy was fixed in glutaraldehyde, embedded in Embed 812 resin, and processed for electron microscopy by standard methods (Malfatti et al, 2013). Ultrathin sections were viewed with Philips CM10 electron microscope (Eindhoven, Netherlands) equipped with Gatan 1k CCD camera.

\section{Antibodies}

The list of the primary antibodies used in this study is provided in Appendix Table S7. The anti- $\alpha$-dystroglycan core protein (a kind donation from S. Kröger) sheep polyclonal antibody was raised against a synthetic peptide corresponding to the carboxy-terminal 20 amino acids of chick $\alpha$-dystroglycan (Herrmann et al, 2000).

\section{Immunohistochemical studies}

Unfixed frozen muscle sections were incubated with primary antibodies overnight at $4{ }^{\circ} \mathrm{C}$, and then with the appropriate secondary antibodies for $30 \mathrm{~min}$.
Cultured myoblasts were permeabilized in $0.2 \%$ Triton X-100 for $10 \mathrm{~min}$ and incubated in $1 \% \mathrm{BSA} / \mathrm{PBS}$ for $45 \mathrm{~min}$; frozen sections were incubated in $5 \%$ normal goat serum/PBS for $45 \mathrm{~min}$. Primary antibodies were used as described in Appendix Table S7 and incubated for $12 \mathrm{~h}$ at $4^{\circ} \mathrm{C}$. After washing, samples were incubated with the appropriate secondary antibodies for $1 \mathrm{~h}$.

PAX7 immunohistochemistry was carried out according to an antigen retrieval protocol, modified from Song et al (2012). Briefly, samples were fixed with $4 \%$ paraformaldehyde, $\mathrm{pH}=7.4$ (PFA) for 15 min. Sections were then immersed in citrate buffer $(\mathrm{pH} \mathrm{6)}$ for $30 \mathrm{~min}$ at $80^{\circ} \mathrm{C}$ and washed with PBS. Afterward, tissue samples were blocked with non-fat milk at $2 \%$ in PBS for $30 \mathrm{~min}$ and washed with PBS. Next, tissue sample were incubated with primary antibody for 2 days and secondary antibody for $2 \mathrm{~h}$ in blocking solution (5\% BSA, $0.5 \%$ Triton X-100 in PBS). $\alpha$ DG-CORE immunohistochemistry required incubation with both biotinylated secondary antibody for $30 \mathrm{~min}$ and streptavidin conjugated to Cy3 for $15 \mathrm{~min}$ in PBS. Finally, the nuclei were stained for $20 \mathrm{~min}$ with To-pro-3-Iodide (Topro) at 1:1,000 in PBS and the slides were coverslipped with fluorescence mounting medium (Dako). The images were acquired on a Zeiss LSM 710 confocal laser scanning microscope. Maximal projections of Z-stacked images were obtained and analyzed with ImageJ software.

\section{Glycoprotein enrichment and Western blot analysis}

Frozen muscle samples and fresh skin fibroblasts were homogenized in RIPA buffer (20 mM Tris- $\mathrm{HCl}$ pH 7.4, $150 \mathrm{mM} \mathrm{NaCl}, 1 \mathrm{mM}$ EDTA, $1 \%$ IGEPAL, $0.1 \%$ SDS) containing protease inhibitor mixture. Western blots of glycoproteins were enriched with wheatgerm agglutinin (WGA) agarose (Sigma-Aldrich) as described previously (Michele et al, 2002). Equivalent amounts of protein lysates non-incubated or incubated with WGA agarose beads were resolved on $7-10 \%$ SDS-PAGE gels and transferred to PDVF membranes (Millipore). Immunoreactivity was detected with secondary antibodies conjugated to horseradish peroxidase (Jackson Immuno Research) and developed with SuperSignal West Femto (Thermo Scientific) using an ImageQuant LAS 4000 MiniGold System (GE Healthcare).

\section{Ligand overlay assay}

Ligand overlay assays in muscle and skin fibroblasts were performed as previously described with minor modifications (Michele et al, 2002; Willer et al, 2012). Briefly, PVDF membranes were incubated with Engelbreth-Holm-Swarm laminin (SigmaAldrich) or agrin (R\&D Systems), overnight at $4^{\circ} \mathrm{C}$ in lamininbinding buffer. Then, membranes were washed and incubated with anti-agrin (Millipore) or anti-laminin (Sigma-Aldrich) primary antibodies and their corresponding secondary antibodies. Blots were imaged using the protocol described for Western blots.

\section{Flow cytometry}

The following method was modified from Stevens et al (2013b). Briefly, an anti- $\alpha$-dystroglycan antibody IIH6 was used to assess the amount of $\alpha$-dystroglycan glycosylation in fibroblasts from patients (II.4 and II.5) and two controls. Fibroblasts (passages 2-3) were 
grown until approximately $90 \%$ confluent. Cells were detached using Accutase (Sigma, UK), centrifuged for $3 \mathrm{~min}$ at $500 \mathrm{~g}$, and counted and resuspended in PBS to a final density of 200,000 fibroblasts per milliliter, centrifuged at 3,000 $\mathrm{g}$ for $3 \mathrm{~min}$, and incubated on ice with IIH6 for $30 \mathrm{~min}$, anti-mouse biotinylated IgM (Vector Labs, USA) for $20 \mathrm{~min}$, and streptavidin-PE (BD Pharmingen, UK) for $15 \mathrm{~min}$. After washing, cells were resuspended in $500 \mathrm{ml}$ of PBS and transferred to FACS tubes (Corning Science, Mex). Data were acquired using the BD FACS Canto II analyzer and analyzed using the BD FACSDiva software (BD Bioscience, US). Two separate controls for each fibroblast population were used: one without any staining to remove background and another without the primary antibody to gate the IIH6-positive population. A total of 50,000 cells were analyzed per experiment. We assessed the percentage of IIH6positive cells as well as the level of IIH6 the mean fluorescence intensity (MFI).

\section{Genetic mapping}

Genomewide genotyping using the Illumina Infinium HumanOmni1Quad, v1.0 BeadChip (Illumina, San Diego, CA, USA) was performed in the DNA samples of the four affected siblings (II.1, II.2, II.4, and II.5) and the unaffected sister (II.3), thus allowing the analysis of over one million assays for each sample. The chips were scanned, and data were loaded into Illumina Genome Studio V2009.1 software. Genome Viewer tool was used to visualize genomic copy number variation as well as individual genotypes. Log $\mathrm{R}$ ratio and $\mathrm{B}$ allele frequency, plotted along the entire genome for all SNPs on the array, were used to analyze all variants. CNV partition 2.4.4 was employed to automatically detect copy number variants as well as regions of extended homozygosity $>500 \mathrm{~kb}$ across the genome. Genotype success rates $>99.7 \%$ were obtained for all assays (Appendix Fig S6).

\section{Exome sequencing}

The capture and subsequent sequencing of exonic regions of DNA samples II.3, II.4, and II.5 was performed at Otogenetics Co (Norcross, GA, USA) using the solution-based SeqCap EZ Human Exome Library v2.0 (Roche NimbleGen, Madison, WI, USA) and the Illumina HiSeq2000, with 100 paired-end runs. An average coverage of $30 \times$ across the exome was achieved for all samples. Reads were aligned to the reference genome hg19 with DNAnexus software (Palo Alto, CA, USA) using default parameters. Single nucleotide polymorphisms and indels were identified and realigned using the Genome Analysis Toolkit (GATK). In order to discard common variants, genetic variations were further verified in the Database of Single Nucleotide Polymorphisms (dbSNP Build ID: 137; http://www.ncbi.nlm.nih.gov/SNP/), the exome variant server (EVS) of the National Heart, Lung, and Blood Institute GO Exome Sequencing Project (Seattle, WA, USA; http://evs.gs. washington.edu/EVS/), and the 1000 Genomes Project (http:// www.1000genomes.org/).

\section{Segregation analysis}

Segregation analysis was performed by means of Sanger sequencing using the primers presented in Appendix Table S4. Cycle sequencing was performed using the Dye Terminator Sequencing Kit (Applied Biosystems, Foster City, CA) and run on an ABI 3100 genetic analyzer. Sequence chromatograms were analyzed using the Sequencher software (Genecodes, Ann Arbor, MI).

\section{Genotyping}

Genotyping of the p.D233E mutation in POGLUT1 gene in the Spanish and the Human Genome Diversity Project series was carried out by real-time PCR using a custom TaqMan ${ }^{\circledR}$ Genomic Assay (Applied Biosystems, Foster City, CA) with appropriate primers and probes (Appendix Table S5). Thermal cycling and end-point polymerase chain reaction (PCR) analysis was performed on a real-time PCR instrument (ABI 7900HT, Applied Biosystems, Foster City, CA). DNA from family members II.3 and II.4 was used as positive controls in all experiments.

\section{POGLUT1 subcloning and mutagenesis}

Human POGLUT1 cDNA cloned into the pEZ-M02 vector was obtained from GeneCopoeia (Rockville, MD, USA). The p.D233E mutation was introduced with the QuikChange II site-directed mutagenesis kit (Agilent Technologies, Santa Clara, CA, USA), as per manufacturer's indications, and confirmed by direct sequencing. The oligonucleotide sequences used for site-directed mutagenesis and Sanger sequencing were as follows: POGLUT1 $5^{\prime}$-CAGCCTCCG

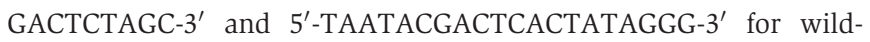
type and POGLUT1 $5^{\prime}$-TCTCGGAAAAACCCAAAACTTGTTGAGGC AGAATACACCAAA- $3^{\prime}$ and $5^{\prime}$-TTTGGTGTATTCTGCCTCAACAAGTT TTGGGTTTTTCCGAGA-3' (mismatched nucleotide to the reference sequence is underlined) for the D233E mutant. cDNAs encoding the luminal domains of wild type and D233E mutant of human POGLUT1, starting from Arg24, were amplified by PCR using the primers, 5'-ATATATAAGCTTCACGCCAGAAGGAGTCAGGTT-3' and 5'-ATCTAGCTCGAGCTAGTTCAGTTTTCAACATTTTGGG-3', and subcloned into a pSecTag2c vector (Invitrogen) in frame with a C-terminal Myc/6xHis tag for expression in mammalian cells. The expression constructs for wild-type human POGLUT1 in pcDNA4 or pTracer vector were also used as a template for site-directed mutagenesis to introduce the D233E mutation (Rana et al, 2011; Takeuchi et al, 2012). The sequences of the primers are $5^{\prime}$-CT TGTTGAGGCAGAATACACCAAAAACCAGGCCTG-3' and 5'-GTATT CTGCCTCAACAAGTTTGGGTTTTTCCGAGACAG-3'. The pcDNA4based construct encodes the recombinant protein with a C-terminal Myc/6xHis tag. The pTracer-based construct encodes the recombinant protein with a FLAG tag, which is immediately downstream of the KTEL sequence at the C-terminus. Successful incorporation of the mutation was confirmed by direct DNA sequencing.

\section{In vitro glycosylation assays}

Myc/6xHis-tagged POGLUT1 proteins were expressed in HEK293T cells by transient transfection, and the secreted proteins were purified from the culture media using Ni-NTA agarose (QIAGEN). POGLUT/POXYLT assays were performed as previously described (Fernandez-Valdivia et al, 2011). Briefly, a $10 \mu \mathrm{l}$ standard reaction mixture contained $50 \mathrm{mM}$ HEPES pH $6.8,10 \mathrm{mM} \mathrm{MnCl}_{2}$, the indicated amounts of acceptor substrates, $0.16 \mu \mathrm{M}$ UDP-[6- $\left.{ }^{3} \mathrm{H}\right]$ glucose 
(2.22 TBq/mmol), $10 \mu \mathrm{M}$ UDP-glucose, $0.5 \%$ Nonidet P-40, and purified proteins. For POXYLT assay, $10 \mu \mathrm{M}$ UDP- $\left[{ }^{14} \mathrm{C}(\mathrm{U})\right]$ xylose (5.43 GBq/mmol) was used as donor substrate. The reaction was performed at $37^{\circ} \mathrm{C}$ for $20 \mathrm{~min}$ and stopped by adding $900 \mu \mathrm{l}$ of 100 mM EDTA pH 8.0. The sample was loaded onto a C18 cartridge (100 mg, Agilent Technologies). After the cartridge was washed with $5 \mathrm{ml}$ of $\mathrm{H}_{2} \mathrm{O}$, the EGF repeat was eluted with $1 \mathrm{ml}$ of $80 \%$ methanol. Incorporation of $\left[6-{ }^{3} \mathrm{H}\right]$ glucose or $\left[{ }^{14} \mathrm{C}(\mathrm{U})\right]$ xylose into the EGF repeats was determined by scintillation counting of the eluate. Reactions without substrates were used as background control. Data are from three independent assays. The values indicate mean \pm SEM.

$O$-glucosylation or $O$-xylosylation of human factor IX EGF repeat and EGF16 from mouse Notch2 was performed using a modified POGLUT/POXYLT assay. A $100 \mu \mathrm{l}$ reaction mixture contained $50 \mathrm{mM}$ HEPES pH $6.8,10 \mathrm{mM} \mathrm{MnCl}_{2}, 10 \mu \mathrm{M}$ acceptor substrate, $200 \mu \mathrm{M}$ UDP-glucose, and purified wild-type or D233E mutant POGLUT1. For $O$-xylosylation, $200 \mu \mathrm{M}$ UDP-xylose was used as donor substrate. Approximately $500 \mathrm{ng}$ of each recombinant enzyme was used. The reaction was performed at $37^{\circ} \mathrm{C}$ overnight. The reaction products were purified by reverse-phase high-performance liquid chromatography (RP-HPLC, Agilent Technologies, 1200 Series) equipped with a C18 column $(10 \times 250 \mathrm{~mm}$, VYDAC $)$ with a linear gradient of solvent B $(80 \%$ acetonitrile, $0.1 \%$ trifluoroacetic acid (TFA) in water) from 10 to $90 \%$ in solvent A $(0.1 \%$ TFA in water) for $60 \mathrm{~min}$, monitoring absorbance at $214 \mathrm{~nm}$. Elution profiles between 20 and 30 min are shown as milli-absorbance unit (mAU). Peak fractions were pooled, dried down in a Speed-Vac centrifuge, and stored at $-20^{\circ} \mathrm{C}$ for further mass spectral analysis.

\section{Mass spectrometric analysis of glycosylation products}

Mass spectrometric analysis by infusion was performed as described previously (Takeuchi et al, 2012). Briefly, dried samples were resuspended in $0.1 \%$ formic acid in water, spin-filtered, and injected into an Agilent 6340 ion-trap mass spectrometer with a nano-HPLC CHIP-Cube interface at a rate of $18 \mu \mathrm{l} / \mathrm{h}$. The MS peaks for MS/MS were chosen manually, and the data were analyzed using Agilent ChemStation data analysis software. The masses of EGF repeats with different charge states were deconvoluted and shown on the top of peaks in HPLC profiles.

\section{RT-PCR and qRT-PCR analysis}

Total RNA was extracted using QIAzol and RNeasy kit (Qiagen). cDNA was synthesized from $1 \mu \mathrm{g}$ of total RNA using Transcriptor First Strand cDNA Synthesis Kit (Roche). qRT-PCR (100 ng total cDNA per reaction) was performed using TaqMan ${ }^{\circledR}$ Fast Advanced Master Mix (Applied Biosystems) in a 7500 Fast Real-Time PCR System. Relative mRNA levels were compared using the $2^{-\Delta \Delta C_{\mathrm{T}}}$ method, with GAPDH as control (only POGLUT1 mRNA level was relative to desmin). We used TaqMan probes (Applied Biosystems) for human HES1 (Hs00172878_m1), human PAX7 (Hs0024 2962_m1), human POGLUT1 (Hs00220308_m1), human GAPDH (Hs02758991_g1), human DES (Hs00157258_m1), mouse HES1 (Mm01342805_m1), mouse Pax7 (Mm01354484_m1), and mouse GAPDH (Mm03302249_g1).

We selected the disease control muscles from patients with wellcharacterized limb-girdle muscular dystrophies. To avoid possible differences due to the effect of age on the satellite cell population (Chakkalakal et al, 2012; Sousa-Victor et al, 2014), our samples of D233E patients and both healthy and disease controls were agematched (Appendix Table S6).

\section{Generation of the transgenic fly}

To generate transgenic flies capable of overexpressing human POGLUT1 ${ }^{\text {D233E }}$, a BamHI-XbaI fragment containing the POGLUT1 ${ }^{\text {D233E }}$ ORF was released from pTracer-POGLUT1 ${ }^{\text {D233E }}$ FLAG and cloned into the BglII-XbaI sites of pUASTattB (Bischof et al, 2007) to generate pUASTattB-POGLUT1 ${ }^{\text {D233E }}$-FLAG. Generation of pUASTattB-POGLUT $1^{\text {wt }}$-FLAG was described previously (Fernandez-Valdivia et al, 2011). Both constructs were integrated into the VK22 docking site by using $\Phi$ C31-mediated transgenesis to generate UASattB-POGLUT1 ${ }^{\text {D233E }}$-FLAG.VK22 and UASattBPOGLUT1 ${ }^{W T}$-FLAG.VK22 transgenes. Correct integration events were verified by att PCRs, as described previously (Venken et al, 2006).

\section{Analysis of muscle phenotype in Drosophila}

We assessed the effect of POGLUT1 activity toward the development of indirect flight muscles in Drosophila (Gildor et al, 2012). Rescue experiments were performed with fly transgenes expressing human POGLUT1 $^{w t}$ (wild type) and human POGLUT1 ${ }^{\text {D233E }} \cdot w^{1118}$ (wt), rumi $^{79 / 79}$, Mef2-GAL4/UASattB-POGLUT1 ${ }^{\text {WT }}$-FLAG; rumi ${ }^{79 / 79}$, and Mef2-GAL4/UASattB-POGLUT ${ }^{\text {D233E }}$-FLAG; rumi ${ }^{79 / 79}$ animals were raised at $18^{\circ} \mathrm{C}$ until puparium formation. Pupae $0-1 \mathrm{~h}$ after puparium formation (APF) were incubated at indicated temperatures until $25 \%$ pupal development. Pupal indirect flight muscles were dissected and stained using standard methods. Antibodies are rabbit $\alpha$-Twist 1:5,000 (Roth et al, 1989), mouse 22C10 1:50 (DSHB), mouse $\alpha$-FLAG M2 1:100 (Sigma), donkey- $\alpha$-rabbit-Cy3 1:500, donkey- $\alpha$-mouse-Cy5 1:500 (Jackson ImmunoResearch Laboratories). Confocal images were scanned using a Leica TCS-SP5 microscope and processed with Amira5.2.2. Myotube lengths were measured using a two-dimensional measurement tool in Amira5.2.2. Mean myotube lengths and standard deviations were calculated for rumi $i^{79 / 79}$ pupae with no rescue and $r u m i^{79 / 79}$ pupae overexpressing POGLUT1 $^{W T}(n=22)$ or POGLUT1 ${ }^{\text {D233E }}(n=54)$. One-way ANOVA with Tukey's multiple comparisons test was used to determine the $P$-values. Images were processed with Adobe Photoshop CS5; figures were assembled in Adobe Illustrator CS5.

\section{C2C12 cell line culture and Notch inhibition assay}

$\mathrm{C} 2 \mathrm{C} 12$ cell line was cultured in monolayer. The culture media for the $\mathrm{C} 2 \mathrm{C} 12$ myoblast stage contains Dulbecco's minimal essential medium (DMEM, Gibco, BRL, Bethesda, MD), supplemented with $5 \%$ glutamine, $10 \%$ fetal bovine serum, $1 \%$ penicillin-streptomycin-fungizone, and $23 \mathrm{mM}$ HEPES. The cultures were examined regularly to avoid overgrowth. For the Notch inhibition assay, we treated C2C12 myoblasts at $80-90 \%$ confluence with DAPT (SigmaAldrich, USA), a $\gamma$-secretase inhibitor, and a well-established inhibitor of Notch, a $\gamma$-secretase substrate, as previously published (Kuang et al, 2007). We also treated $\mathrm{C} 2 \mathrm{C} 12$ myoblasts with LY3039478 (ApexBio Technology LLC), a more specific and potent Notch inhibitor. For this experiment, culture proliferation and 
differentiation medium were modified by adding $50 \mu \mathrm{M}$ DAPT or $25 \mu \mathrm{M}$ LY3039478, and 50-25 $\mu \mathrm{M}$ DMSO for controls, respectively.

\section{Primary cell culture}

Muscle biopsies from two patients (II.4 and II.5), three age-matched healthy controls and three age-matched disease controls were used for the experiments. Muscle biopsies were minced and cultured in a monolayer. Briefly, the culture medium for the myoblast stage contains 75\% Dulbecco's minimal essential medium (Invitrogen) and 25\% M199 medium (Invitrogen), supplemented with 10\% fetal bovine serum (FBS), $10 \mu \mathrm{g} / \mathrm{ml}$ insulin, $2 \mathrm{mM}$ glutamine, 100 units $/ \mathrm{ml}$ penicillin, $100 \mu \mathrm{g} / \mathrm{ml}$ streptomycin, $0.25 \mu \mathrm{g} / \mathrm{ml}$ fungizone, $10 \mathrm{ng} / \mathrm{ml}$ epidermal growth factor, and $25 \mathrm{ng} / \mathrm{ml}$ fibroblast growth factor. To obtain highly purified myoblasts, each $10^{7}$ cells were mixed with $20 \mu \mathrm{l}$ of CD56-coated microbeads (Miltenyi Biotec, Bergisch Gladbach, Germany) and incubated at $6-12^{\circ} \mathrm{C}$ for $15 \mathrm{~min}$. Unbound microbeads were removed by washing cells in excess PBS buffer followed by centrifugation at $300 \times \mathrm{g}$ for $10 \mathrm{~min}$. The cell pellet was resuspended in PBS buffer to a concentration of $2 \times 10^{8}$ cells $/ \mathrm{ml}$ before separation on a midiMACS cell separator (Miltenyi Biotec, Bergisch Gladbach, Germany). CD56-positive cells were seeded at 13,000 cells $/ \mathrm{cm}^{2}$ using the culture medium for the myoblast stage containing $15 \%$ of FBS and without growth factors to avoid interference with the results. We examine proliferation at different days. When the myoblasts started to fuse, the medium was substituted with one containing $2 \%$ of FBS. We measured the fusion index 4 days after medium change by calculating the mean percentage of nuclei in myotubes in respect to the total number of nuclei (myoblasts + myotubes).

Human skin biopsies were minced and cultured. Fibroblasts were grown in DMEM containing $15 \%$ fetal bovine serum and $1 \%$ penicillin-streptomycin-fungizone at $37^{\circ} \mathrm{C}$ with $5 \% \mathrm{CO}_{2}$.

\section{Myoblast immortalization}

Myoblasts from II.4 patient and healthy control were transduced with lentiviral vectors encoding hTERT and cdk4 containing puromycin and neomycin selection markers, respectively. Transduced cells were selected with puromycin $(1 \mu \mathrm{g} / \mathrm{ml})$ for 6 days and neomycin $(1 \mathrm{mg} / \mathrm{ml})$ for 10 days. Infected cells were purified using an immunomagnetic cell sorting system (MACS; Miltenyi) according to the manufacturer's instructions with anti-CD56 microbeads. Cells were seeded at clonal density, and individual myogenic clones were isolated and characterized.

\section{Fibroblast myogenic conversion}

Fibroblasts from II.4 patient and healthy control were transduced with lentiviral vector encoding hTert. Transduced cells were selected with puromycin $(1 \mu \mathrm{g} / \mathrm{ml})$ for 6 days. Immortalized fibroblasts were then transduced with a lentiviral vector encoding inducible MyoD. For MyoD induction, doxycycline $(2 \mu \mathrm{g} / \mathrm{ml})$ was added in the proliferation medium (this day was considered day 0 ).

\section{Lentivirus generation and infection}

We generated lentiviral vectors (LV), based on the calcium phosphate method as previously described (Yoshida et al, 2013). Briefly,
HEK293T cells were transfected using transfer plasmids, EF.hICN1.CMV.GFP (\#17623, Addgene) or EF.CMV.GFP, together with psPAX2 packaging and pMD2.G envelope plasmid DNA at a ratio of $4: 3: 1$, respectively. Then, $48 \mathrm{~h}$ post-transfection, medium was collected, filtered, and concentrated by centrifugation for 90 min at 105,000 × g using a SW 32 Ti Beckman rotor. Supernatant was completely removed and pellet diluted in $100 \mu$ PBS overnight at $4^{\circ} \mathrm{C}$, titered by FACS, and stored at $-80^{\circ} \mathrm{C}$ until use. Immortalized myoblasts from patients were infected with LV-NICD-GFP or LVGFP, and immortalized myoblasts from control with LV-GFP. After 7 days, GFP-positive cells were purified by FACS.

\section{Study approval}

This study was approved by the Institutional Research Ethic Committee at Hospital Universitario Virgen del Rocío in Sevilla (Spain). Written informed consent was received from participants, prior to inclusion in the study, for genetic studies, for muscle and skin biopsies, and for pictures appearing in the manuscript.

Expanded View for this article is available online.

\section{Acknowledgements}

We thank Isabel IIla (Hospital Santa Creu i Sant Pau) and Salvatore DiMauro (Columbia University) for their critical comments; Montserrat Olivé (Hospital de Bellvitge) for her supporting on muscle imaging; Cristina Dominguez (Hospital 12 Octubre) for ceding us muscle samples; and Cloria Cantero for helping with quantifications. Stephan Kröger (Münich University) for kind donation of the antibody against the $\alpha$-dystroglycan core (clone no. 317); Maria Leptin (Cologne University) for anti-Twist antibody; David Comas (Institut de Biología Evolutiva, Pompeu Fabra University), for providing DNA samples; Developmental Studies Hybridoma Bank for 22C10 antibody; Bloomington Drosophila stock center (NIH P400D018537) for the Mef2-CAL4 strain; Confocal Microscopy Core of the BCM IDDRC (1U54HD083092; the Eunice Kennedy NICHD); Platform for immortalization of human cells of the Myology Institute in Paris; and Raquel Gómez (Biomedicine Institute of Seville Pathology Core Facility), Juan J. PérezMoreno (Centro Andaluz de Biología del Desarrollo), and O. Akman (Columbia University) for their technical support. Supported in part by grants from the Health Institute Carlos III and FEDER (FIS PI10/02410, PI13-01739, and BA12-00097 to C. Paradas, FIS12/2291 to E. Gallardo, and CIBERNED to R. Fernández-Chacón), the Andalusian Government (PI-00172014 to C. Paradas and P12-CTS-2232 to R. Fernández-Chacón), The Matsumae International Foundation (to C. Paradas), the NIH/NIGMS (R01GM061126 to R.S. Haltiwanger and R01GM084135 to H. Jafar-Nejad), the NIH (U54 NS078059 from NINDS and NICHD, R01 HD056103 from NICHD to M. Hirano), and the MDA USA (to M. Hirano).

\section{Author contributions}

$\mathrm{CP}$ and JC designed the study. ES-M performed $\alpha$-dystroglycan expression and function studies, cell culture, satellite cells, and myogenesis analysis. HT performed biochemical in vitro assays. TVL and BE performed the fly studies. $\mathrm{CP}, \mathrm{MC}-\mathrm{S}, \mathrm{YM}$, and $\mathrm{CM}$ handled patients and collected biological samples from the patients. ER and ES-M processed and studied muscle biopsy. CP, MC-S, and JAM-L analyzed the radiological findings. FM, CP, JLN-G, and LG-S analyzed protein expression and mRNA levels. JC and GP performed and genetic studies. MCR and XS-C performed cell culture. AB generated the immortalized cells. EA-G, EG, and RF-C provided critical discussion on the 


\section{The paper explained}

\section{Problem}

The fact that in a significant number of patients suffering from a muscular dystrophy, the responsible gene is still unknown stimulates research to describe new molecular and cellular pathomechanisms underlying muscle degeneration. It is known that even in healthy individuals, common daily activities cause skeletal muscle lesions that are physiologically repaired through activation of satellite cells. Accordingly, it is logical to envision a scenario where alteration in the satellite cell homeostasis could lead to muscle fiber degeneration because of accumulation of unrepaired damage. Indeed, this appears to be the case with the family we present carrying a homozygous missense D233E mutation in POGLUT1 gene: a muscular dystrophy as a consequence of defective muscle regeneration.

\section{Results}

It is widely known that Notch signaling pathway plays critical roles in the highly coordinated muscle regenerative process, maintaining an appropriate population of satellite cells and preventing premature differentiation. Our previous work in Drosophila and mammalian cell lines has shown that the addition of $O$-linked glucose to Notch receptors by protein O-glucosyltransferase 1 (POGLUT1; also known as Rumi) is required for Notch signaling. In the investigated family, a homozygous missense D233E mutation in POGLUT1 dramatically reduces its enzymatic activity on Notch. As a result, the investigated family shows a defect in Notch signaling with a significant depletion of satellite cells, resulting in defective muscle regeneration and ultimately leading to muscle dystrophy. In addition, primary myoblasts from our patients displayed reduced selfrenewal capacity and enhanced differentiation in culture, as expected under a Notch signaling defective environment. We show that the enzymatic activity of POGLUT1 is also essential for the formation of adult muscles by myoblasts in Drosophila. Moreover, cross-species overexpression studies in Drosophila indicate that the D233E mutation impairs the ability of human POGLUT1 in rescuing the muscle defects in flies lacking endogenous POGLUT1 activity. We have previously shown that complete loss of POGLUT1 results in embryonic lethality, but our patients only exhibit an adult-onset muscular dystrophy. Our data provide a clear explanation for this apparent discrepancy: First, the D233E does not affect the expression level and the subcellular localization of POGLUT1, and second, despite the severe decrease in the enzymatic activity of POGLUT1 ${ }^{\text {D233E }}$, this mutant protein maintains a residual activity. Of note, our patients also exhibited a muscle-specific reduction in glycosylation and laminin binding of $\alpha$-dystroglycan. While these defects probably contribute to muscle dystrophy in our patients, we do not favor the hypothesis that they constitute the primary underlying mechanism for the disease, as $\alpha$-dystroglycan lacks POCLUT1 target sites and patient fibroblasts show normal $\alpha$-dystroglycan glycosylation and laminin binding. Instead, we propose that altered proliferation and differentiation of satellite cells due to decreased Notch signaling ultimately alter the $\alpha$-dystroglycan glycosylation, which is a dynamic process during muscle differentiation.

\section{Impact}

Our data link a missense mutation in POGLUT1 to a novel form of muscular dystrophy and suggest that Notch-dependent loss of satellite cells is the primary pathomechanism for the disease in our patients. The muscle-specific phenotypes in our patients indicate that muscle stem cells are much more sensitive to a decrease in POGLUT1 enzymatic activity compared with other human cell types and suggest that small molecules affecting the activity of POGLUT1 in muscle could be of potential benefit in controlling the behavior of satellite cells, paving the way for potential future therapies. research. $\mathrm{CP}, \mathrm{MH}, \mathrm{RSH}$, and $\mathrm{HJ}-\mathrm{N}$ supervised and mentored all work. $\mathrm{CP}$ coordinated all the study and wrote the initial manuscript. All authors contributed to the final version of the manuscript.

\section{Conflict of interest}

The authors declare that they have no conflict of interest.

\section{References}

Acar M, Jafar-Nejad H, Takeuchi H, Rajan A, Ibrani D, Rana NA, Pan H, Haltiwanger RS, Bellen HJ (2008) Rumi is a CAP10 domain glycosyltransferase that modifies Notch and is required for Notch signaling. Cell 132: 247-258

Awano H, Blaeser A, Wu B, Lu P, Keramaris-Vrantsis E, Lu Q (2015) Dystroglycanopathy muscles lacking functional glycosylation of alphadystroglycan retain regeneration capacity. Neuromuscul Disord 25: $474-484$

Barresi R, Campbell KP (2006) Dystroglycan: from biosynthesis to pathogenesis of human disease. J Cell Sci 119: 199-207

Basmanav FB, Oprisoreanu AM, Pasternack SM, Thiele H, Fritz G, Wenzel J, Grosser L, Wehner M, Wolf S, Fagerberg C et al (2014) Mutations in POGLUT1, encoding protein O-glucosyltransferase 1 , cause autosomal-dominant dowling-degos disease. Am J Hum Genet 94: $135-143$

Bischof J, Maeda RK, Hediger M, Karch F, Basler K (2007) An optimized transgenesis system for Drosophila using germ-line-specific phiC31 integrases. Proc Natl Acad Sci USA 104: 3312-3317

Bjornson CR, Cheung TH, Liu L, Tripathi PV, Steeper KM, Rando TA (2012) Notch signaling is necessary to maintain quiescence in adult muscle stem cells. Stem Cells 30: 232-242

Bonnemann CG, Wang CH, Quijano-Roy S, Deconinck N, Bertini E, Ferreiro A, Muntoni F, Sewry C, Beroud C, Mathews KD et al (2014) Diagnostic approach to the congenital muscular dystrophies. Neuromuscul Disord 24: 289-311

Brohl D, Vasyutina E, Czajkowski MT, Griger J, Rassek C, Rahn HP, Purfurst B, Wende H, Birchmeier C (2012) Colonization of the satellite cell niche by skeletal muscle progenitor cells depends on Notch signals. Deu Cell 23: $469-481$

Carss KJ, Stevens E, Foley AR, Cirak S, Riemersma M, Torelli S, Hoischen A, Willer T, van Scherpenzeel M, Moore SA et al (2013) Mutations in GDPmannose pyrophosphorylase $B$ cause congenital and limb-girdle muscular dystrophies associated with hypoglycosylation of alpha-dystroglycan. Am J Hum Genet 93: 29-41

Chakkalakal JV, Jones KM, Basson MA, Brack AS (2012) The aged niche disrupts muscle stem cell quiescence. Nature 490: $355-360$

Chandrasekharan K, Martin PT (2010) Genetic defects in muscular dystrophy. Methods Enzymol 479: 291-322

Choi J, Costa ML, Mermelstein CS, Chagas C, Holtzer S, Holtzer H (1990) MyoD converts primary dermal fibroblasts, chondroblasts, smooth muscle, and retinal pigmented epithelial cells into striated mononucleated myoblasts and multinucleated myotubes. Proc Natl Acad Sci USA 87: 7988-7992

Christian JL (2000) BMP, Wnt and Hedgehog signals: how far can they go? Curr Opin Cell Biol 12: $244-249$

Cirak S, Foley AR, Herrmann R, Willer T, Yau S, Stevens E, Torelli S, Brodd L, Kamynina A, Vondracek P et al (2013) ISPD gene mutations are a common cause of congenital and limb-girdle muscular dystrophies. Brain 136: 269-281 
Cohn RD, Henry MD, Michele DE, Barresi R, Saito F, Moore SA, Flanagan JD, Skwarchuk MW, Robbins ME, Mendell JR et al (2002) Disruption of DAG1 in differentiated skeletal muscle reveals a role for dystroglycan in muscle regeneration. Cell 110: 639-648

Collins CA, Olsen I, Zammit PS, Heslop L, Petrie A, Partridge TA, Morgan JE (2005) Stem cell function, self-renewal, and behavioral heterogeneity of cells from the adult muscle satellite cell niche. Cell 122: 289-301

Conboy IM, Rando TA (2002) The regulation of Notch signaling controls satellite cell activation and cell fate determination in postnatal myogenesis. Deu Cell 3: $397-409$

Cote PD, Moukhles H, Lindenbaum M, Carbonetto S (1999) Chimaeric mice deficient in dystroglycans develop muscular dystrophy and have disrupted myoneural synapses. Nat Genet 23: 338-342

Ervasti JM, Ohlendieck K, Kahl SD, Gaver MG, Campbell KP (1990) Deficiency of a glycoprotein component of the dystrophin complex in dystrophic muscle. Nature 345: 315-319

Ervasti JM, Campbell KP (1991) Membrane organization of the dystrophinglycoprotein complex. Cell 66: 1121-1131

Ervasti JM, Campbell KP (1993) A role for the dystrophin-glycoprotein complex as a transmembrane linker between laminin and actin. J Cell Biol 122: $809-823$

Fernandez-Valdivia R, Takeuchi H, Samarghandi A, Lopez M, Leonardi J, Haltiwanger RS, Jafar-Nejad H (2011) Regulation of mammalian Notch signaling and embryonic development by the protein Oglucosyltransferase Rumi. Development 138: 1925-1934

Freeze HH, Chong JX, Bamshad MJ, Ng BC (2014) Solving glycosylation disorders: fundamental approaches reveal complicated pathways. Am J Hum Genet 94: 161-175

Gildor B, Schejter ED, Shilo BZ (2012) Bidirectional Notch activation represses fusion competence in swarming adult Drosophila myoblasts. Development 139: $4040-4050$

Goddeeris MM, Wu B, Venzke D, Yoshida-Moriguchi T, Saito F, Matsumura K, Moore SA, Campbell KP (2013) LARGE glycans on dystroglycan function as a tunable matrix scaffold to prevent dystrophy. Nature 503: $136-140$

Haltom AR, Lee TV, Harvey BM, Leonardi J, Chen YJ, Hong Y, Haltiwanger RS, Jafar-Nejad H (2014) The protein O-glucosyltransferase Rumi modifies eyes shut to promote rhabdomere separation in Drosophila. PLoS Genet 10: e1004795

Hara Y, Balci-Hayta B, Yoshida-Moriguchi T, Kanagawa M, Beltran-Valero de Bernabe D, Gundesli H, Willer T, Satz JS, Crawford RW, Burden SJ et al (2011) A dystroglycan mutation associated with limb-girdle muscular dystrophy. N Engl J Med 364: 939-946

Hase S, Kawabata S, Nishimura H, Takeya H, Sueyoshi T, Miyata T, Iwanaga S, Takao T, Shimonishi Y, Ikenaka T (1988) A new trisaccharide sugar chain linked to a serine residue in bovine blood coagulation factors VII and IX. J Biochem 104: $867-868$

Henry MD, Campbell KP (1998) A role for dystroglycan in basement membrane assembly. Cell 95: 859-870

Herrmann R, Straub V, Blank M, Kutzick C, Franke N, Jacob EN, Lenard HG, Kroger S, Voit T (2000) Dissociation of the dystroglycan complex in caveolin-3-deficient limb girdle muscular dystrophy. Hum Mol Genet 9: $2335-2340$

Hori K, Sen A, Artavanis-Tsakonas S (2013) Notch signaling at a glance. J Cell Sci 126: $2135-2140$

Inamori K, Yoshida-Moriguchi T, Hara Y, Anderson ME, Yu L, Campbell KP (2012) Dystroglycan function requires xylosyl- and glucuronyltransferase activities of LARGE. Science 335: 93-96
Jafar-Nejad H, Leonardi J, Fernandez-Valdivia R (2010) Role of glycans and glycosyltransferases in the regulation of Notch signaling. Glycobiology 20: 931-949

Johnston SH, Rauskolb C, Wilson R, Prabhakaran B, Irvine KD, Vogt TF (1997) A family of mammalian Fringe genes implicated in boundary determination and the Notch pathway. Development 124: 2245-2254

Kanagawa M, Kobayashi K, Tajiri M, Manya H, Kuga A, Yamaguchi Y, AkasakaManya K, Furukawa J, Mizuno M, Kawakami H et al (2016) Identification of a post-translational modification with ribitol-phosphate and its defect in muscular dystrophy. Cell Rep 14: 2209-2223

Kuang S, Kuroda K, Le Grand F, Rudnicki MA (2007) Asymmetric self-renewal and commitment of satellite stem cells in muscle. Cell 129: 999-1010

Lee TV, Sethi MK, Leonardi J, Rana NA, Buettner FF, Haltiwanger RS, Bakker H, Jafar-Nejad H (2013) Negative regulation of notch signaling by xylose. PLoS Genet 9: e1003547

Leonardi J, Fernandez-Valdivia R, Li YD, Simcox AA, Jafar-Nejad H (2011) Multiple O-glucosylation sites on Notch function as a buffer against temperature-dependent loss of signaling. Development 138: 3569-3578

Li M, Cheng R, Liang J, Yan H, Zhang H, Yang L, Li C, Jiao Q, Lu Z, He J et al (2013) Mutations in POFUT1, encoding protein O-fucosyltransferase 1, cause generalized Dowling-Degos disease. Am J Hum Genet 92: 895-903

Lin S, Shen H, Jin B, Gu Y, Chen Z, Cao C, Hu C, Keller C, Pear WS, Wu L (2013) Brief report: blockade of Notch signaling in muscle stem cells causes muscular dystrophic phenotype and impaired muscle regeneration. Stem Cells 31: 823-828

Ma W, Du J, Chu Q, Wang Y, Liu L, Song M, Wang W (2011) hCLP46 regulates U937 cell proliferation via Notch signaling pathway. Biochem Biophys Res Commun 408: 84-88

Malfatti E, Olive M, Taratuto AL, Richard P, Brochier G, Bitoun M, Gueneau L, Laforet P, Stojkovic T, Maisonobe T et al (2013) Skeletal muscle biopsy analysis in reducing body myopathy and other FHL1-related disorders. J Neuropathol Exp Neurol 72: 833-845

Mamchaoui K, Trollet C, Bigot A, Negroni E, Chaouch S, Wolff A, Kandalla PK, Marie S, Di Santo J, St Guily JL et al (2011) Immortalized pathological human myoblasts: towards a universal tool for the study of neuromuscular disorders. Skelet Muscle 1: 34

Manya H, Chiba A, Yoshida A, Wang X, Chiba Y, Jigami Y, Margolis RU, Endo T (2004) Demonstration of mammalian protein O-mannosyltransferase activity: coexpression of POMT1 and POMT2 required for enzymatic activity. Proc Natl Acad Sci USA 101: 500-505

Mauro A (1961) Satellite cell of skeletal muscle fibers. J Biophys Biochem Cytol 9: $493-495$

Michele DE, Barresi R, Kanagawa M, Saito F, Cohn RD, Satz JS, Dollar J, Nishino I, Kelley RI, Somer $\mathrm{H}$ et al (2002) Post-translational disruption of dystroglycan-ligand interactions in congenital muscular dystrophies. Nature 418: 417-422

Moloney DJ, Shair LH, Lu FM, Xia J, Locke R, Matta KL, Haltiwanger RS (2000) Mammalian Notch1 is modified with two unusual forms of O-linked glycosylation found on epidermal growth factor-like modules. J Biol Chem 275: 9604-9611

Mourikis P, Tajbakhsh S (2014) Distinct contextual roles for Notch signalling in skeletal muscle stem cells. BMC Deu Biol 14: 2

Muntoni F, Torelli S, Wells DJ, Brown SC (2011) Muscular dystrophies due to glycosylation defects: diagnosis and therapeutic strategies. Curr Opin Neurol 24: 437-442

Nakamura N, Lyalin D, Panin VM (2010) Protein O-mannosylation in animal development and physiology: from human disorders to Drosophila phenotypes. Semin Cell Deu Biol 21: 622-630 
Okajima T, Xu A, Lei L, Irvine KD (2005) Chaperone activity of protein Ofucosyltransferase 1 promotes notch receptor folding. Science 307 : $1599-1603$

Olguin HC, Olwin BB (2004) Pax-7 up-regulation inhibits myogenesis and cell cycle progression in satellite cells: a potential mechanism for self-renewal. Deu Biol 275: 375-388

Rahimov F, Kunkel LM (2013) The cell biology of disease: cellular and molecular mechanisms underlying muscular dystrophy. J Cell Biol 201: 499-510

Ramkumar N, Harvey BM, Lee JD, Alcorn HL, Silva-Gagliardi NF, McGlade C], Bestor TH, Wijnholds J, Haltiwanger RS, Anderson KV (2015) Protein Oglucosyltransferase 1 (POGLUT1) promotes mouse gastrulation through modification of the apical polarity protein CRUMBS2. PLoS Genet 11: e1005551

Rana NA, Nita-Lazar A, Takeuchi H, Kakuda S, Luther KB, Haltiwanger RS (2011) O-glucose trisaccharide is present at high but variable stoichiometry at multiple sites on mouse Notch1. J Biol Chem 286: 31623-31637

Riemersma M, Froese DS, van Tol W, Engelke UF, Kopec J, van Scherpenzeel M, Ashikov A, Krojer T, von Delft F, Tessari M et al (2015) Human ISPD is a cytidyltransferase required for dystroglycan O-mannosylation. Chem Biol 22: $1643-1652$

Ross J, Benn A, Jonuschies J, Boldrin L, Muntoni F, Hewitt JE, Brown SC, Morgan JE (2012) Defects in glycosylation impair satellite stem cell function and niche composition in the muscles of the dystrophic Large (myd) mouse. Stem Cells 30: 2330-2341

Roth S, Stein D, Nusslein-Volhard C (1989) A gradient of nuclear localization of the dorsal protein determines dorsoventral pattern in the Drosophila embryo. Cell 59: 1189-1202

Sacco A, Doyonnas R, Kraft P, Vitorovic S, Blau HM (2008) Self-renewal and expansion of single transplanted muscle stem cells. Nature 456: 502-506

Song J, Zhong C, Bonaguidi MA, Sun GJ, Hsu D, Gu Y, Meletis K, Huang ZJ, Ge S, Enikolopov $G$ et al (2012) Neuronal circuitry mechanism regulating adult quiescent neural stem-cell fate decision. Nature 489: 150-154

Sousa-Victor P, Gutarra S, Garcia-Prat L, Rodriguez-Ubreva J, Ortet L, RuizBonilla V, Jardi M, Ballestar E, Gonzalez S, Serrano AL et al (2014) Geriatric muscle stem cells switch reversible quiescence into senescence. Nature 506: $316-321$

Stanley P, Okajima T (2010) Roles of glycosylation in Notch signaling. Curr Top Deu Biol 92: 131-164

Stevens E, Carss KJ, Cirak S, Foley AR, Torelli S, Willer T, Tambunan DE, Yau S, Brodd L, Sewry CA et al (2013a) Mutations in B3GALNT2 cause congenital muscular dystrophy and hypoglycosylation of alpha-dystroglycan. Am J Hum Genet 92: 354-365

Stevens E, Torelli S, Feng L, Phadke R, Walter MC, Schneiderat P, Eddaoudi A, Sewry CA, Muntoni F (2013b) Flow cytometry for the analysis of alphadystroglycan glycosylation in fibroblasts from patients with dystroglycanopathies. PLOS ONE 8: e68958

Sun D, Li H, Zolkiewska A (2008) The role of Delta-like 1 shedding in muscle cell self-renewal and differentiation. J Cell Sci 121: 3815-3823

Takeuchi H, Haltiwanger RS (2010) Role of glycosylation of Notch in development. Semin Cell Deu Biol 21: 638-645

Takeuchi H, Fernandez-Valdivia RC, Caswell DS, Nita-Lazar A, Rana NA, Garner TP, Weldeghiorghis TK, Macnaughtan MA, Jafar-Nejad H, Haltiwanger RS (2011) Rumi functions as both a protein Oglucosyltransferase and a protein O-xylosyltransferase. Proc Natl Acad Sci USA 108: $16600-16605$
Takeuchi H, Kantharia J, Sethi MK, Bakker H, Haltiwanger RS (2012) Sitespecific O-glucosylation of the epidermal growth factor-like (EGF) repeats of notch: efficiency of glycosylation is affected by proper folding and amino acid sequence of individual EGF repeats. J Biol Chem 287: $33934-33944$

Takeuchi H, Haltiwanger RS (2014) Significance of glycosylation in Notch signaling. Biochem Biophys Res Commun 453: 235-242

Thakurdas SM, Lopez MF, Kakuda S, Fernandez-Valdivia R, Zarrin-Khameh N, Haltiwanger RS, Jafar-Nejad H (2016) Jagged1 heterozygosity in mice results in a congenital cholangiopathy which is reversed by concomitant deletion of one copy of Poglut1 (Rumi). Hepatology 63: $550-565$

Varki A, Sharon N (2009) Historical background and overview. In Essentials of glycobiology, Varki A, Cummings RD, Esko JD, Freeze HH, Stanley P, Bertozzi CR, Hart GW, Etzler EE (eds), pp 1-22. New York: Cold Spring Harbor Laboratory Press

Venken KJ, He Y, Hoskins RA, Bellen HJ (2006) P[acman]: a BAC transgenic platform for targeted insertion of large DNA fragments in $D$. melanogaster. Science 314: 1747-1751

Vieira NM, Elvers I, Alexander MS, Moreira YB, Eran A, Gomes JP, Marshall JL, Karlsson EK, Verjovski-Almeida S, Lindblad-Toh K et al (2015) Jagged 1 rescues the duchenne muscular dystrophy phenotype. Cell 163: $1204-1213$

Willer T, Prados B, Falcon-Perez JM, Renner-Muller I, Przemeck GK, Lommel M, Coloma A, Valero MC, de Angelis MH, Tanner W et al (2004) Targeted disruption of the Walker-Warburg syndrome gene Pomt1 in mouse results in embryonic lethality. Proc Natl Acad Sci USA 101: $14126-14131$

Willer T, Lee H, Lommel M, Yoshida-Moriguchi T, de Bernabe DB, Venzke D, Cirak S, Schachter H, Vajsar J, Voit T et al (2012) ISPD loss-of-function mutations disrupt dystroglycan 0-mannosylation and cause WalkerWarburg syndrome. Nat Genet 44: 575-580

Willer T, Inamori K, Venzke D, Harvey C, Morgensen G, Hara Y, Beltran Valero de Bernabe D, Yu L, Wright KM, Campbell KP (2014) The glucuronyltransferase B4CAT1 is required for initiation of LARGE-mediated alpha-dystroglycan functional glycosylation. eLife 3: e03941

Yamamoto T, Toyoda C, Kobayashi M, Kondo E, Saito K, Osawa M (1997) Pialglial barrier abnormalities in fetuses with Fukuyama congenital muscular dystrophy. Brain Deu 19: $35-42$

Yan D, Lin X (2009) Shaping morphogen gradients by proteoglycans. Cold Spring Harb Perspect Biol 1: a002493

Yoshida S, Tsutsumi S, Muhlebach C, Sourbier C, Lee MJ, Lee S, Vartholomaiou E, Tatokoro M, Beebe K, Miyajima N et al (2013) Molecular chaperone TRAP1 regulates a metabolic switch between mitochondrial respiration and aerobic glycolysis. Proc Natl Acad Sci USA 110: E1604-E1612

Yoshida-Moriguchi T, Willer T, Anderson ME, Venzke D, Whyte T, Muntoni F, Lee H, Nelson SF, Yu L, Campbell KP (2013) SCK196 is a glycosylationspecific O-mannose kinase required for dystroglycan function. Science 341: $896-899$

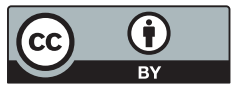

License: This is an open access article under the terms of the Creative Commons Attribution 4.0 License, which permits use, distribution and reproduction in any medium, provided the original work is properly cited. 